\title{
Influence of Internet of Things (IoT) In Association of Data Mining Towards the Development Smart Cities-A Review Analysis
}

\author{
E. B. Priyanka* and S. Thangavel \\ Department of Mechatronics Engineering, Kongu Engineering College, Perundurai, India
}

Received 30 April 2020; Accepted 20 July 2020

\begin{abstract}
The modern wireless sensor network is focused on integrating the emerging technology of IoT with the real world for enhanced data processing to improve automation and communication more smartly and safely. In the present industrial upgradation with IoT application greatly proved its positive influences on the operation and management phases along with progressive adoption of cloud computing and big data analytics. In Industry, smart monitoring and distributed control of entire architecture are hold up with the renovated technology of the Internet of Things (IoT). It lies on the top layer of the wireless communication network to afford enhanced connectivity among smart wireless or wired sensor with the embedded modern controller to the cloud server. In IoT application, real-time data acquisition is done securely and transmitted to the data analytics services to identify any catastrophic situation existence. It is reliable and moved industrial automation to the next step evolution of formulating to make proactive decisions to decide and react to the industrial variation constraints. Though modernization has shifted to analog to the digital generation, it is interoperable with available protocols and data standardizations in industrial sectors. Hence this paper enumerates the detailed configurations and features of IoT application involved in the modern communication platform towards the development of smart cities by incorporating data mining using AI (Artificial Intelligence). Further, the trending overview of Industrial IoT application in risk-based environments with complete knowledge-based architecture with incorporated wireless technology and protocols are outlined.
\end{abstract}

Keywords: IoT, Wireless communication, Protocols, Industrial IoT application.

\section{Introduction}

The next evolution of technology is an IoT paves the way to resolve existing monitoring techniques with reconditioned data communication and hardware networks. The International Telecommunication Union (ITU) revamped the application layer and transport layers to inhibit the feasibility of the Internet of Things. The condition analysis with prerequisites formulated data structure is well practically applicable in the IoT platform. The broadcasting spectrum of $4 \mathrm{~g}$ and $5 \mathrm{G}$ with enhanced communication protocols for secured transmission from the industrial sector to the cloud server is marching towards better development in research areas [1]. The IoT includes processing in both knowledge and technological path by giving the dominant role to the things and semantic analysis conditions through the internet path. The Internet of Things is integrating the real-world to the future pathway of humans with computer programming to better respond to the action when it is monitored exactly with enriched distributed control on the industry [2].

In industrial computerization, the increment in the production rate with reduced maintenance is achieved with the extreme contribution of the Internet of Things due to the state of "monitor and respond". The local control has shifted towards online control with a pre-emptive based decision algorithm with Machine Learning techniques [3]. In real-time computation by the exploring manipulator, the Internet of

*E-mail address: priyankabhaskaran1993@gmail.com ISSN: 1791-2377 @ 2020 School of Science, IHU. All rights reserved. doi:10.25103/jestr.134.01
Things possesses: (1) a collective sympathetic of the analyzing environment of its users and intellectual based embedded sensor systems, (2) software developmental approach and uninterrupted communication networks to data $\log$ in database management where it is pertinent and (3) the analytic outfits aims for self-directed and smart performances [4]. The above three fundamental groundwork helps to tackle the complex environment in a smarter and better-computed way to change the situation to the normal by wireless internet distributed way in industrial sectors. Figure 1 outlines the major focus of IoT development concerning various views of applications [5].

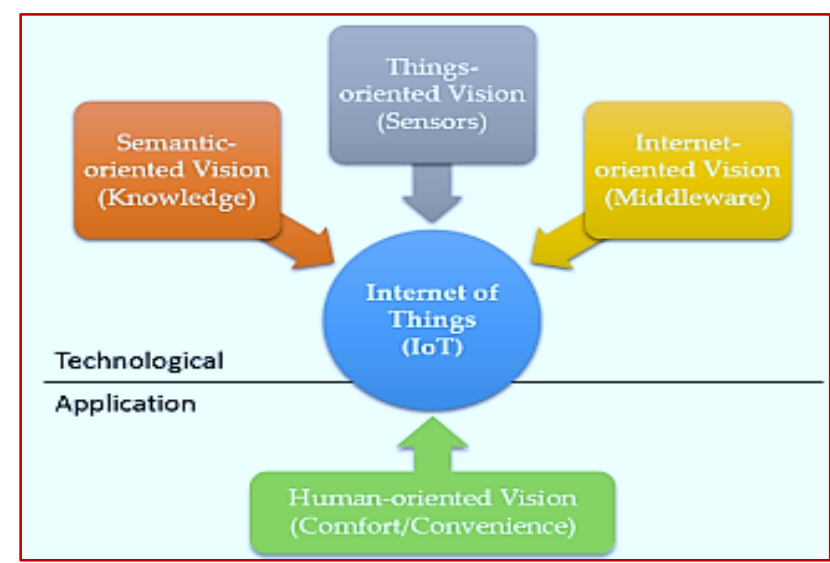

Fig. 1. IoT's basic views concerning an application [2] 
In general, the IoT application in the industrial sectors with a deep core of concentration on data analytics and future forecasting by correlating embedded and wireless sensor systems is given in Figure 2 is a schematic representation.

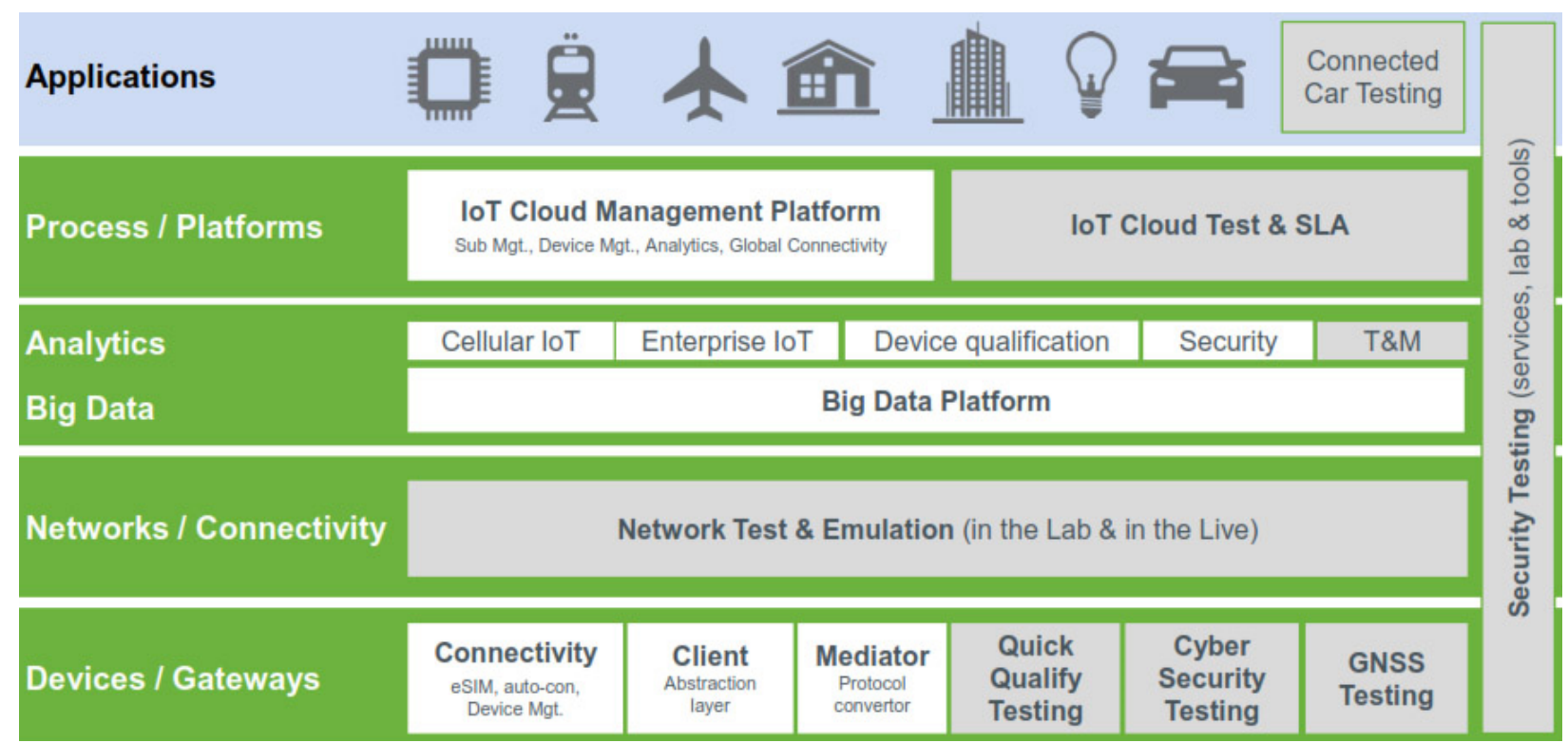

Fig. 2. Application wise involved embedded network system incorporating IoT [5]

Innovative advances and dynamic selection of IoT advances have empowered the improvement of new data and correspondence frameworks - the pervasive data frameworks [6]. An objective of pervasive processing is to interconnect the physical world through the Internet. IoT and Cloud Computing are key empowering innovations to manufacture such data and correspondence frameworks. IoT structures system of items that (1) have one of a kind identifiers, (2) may have the ability to gather information from the earth principally through sensors, (3) work and cooperate with the physical world by handling gathered information, and (4) utilize the Internet measures to impart and examine the information for further administrations. Since the workplace in a larger part of the industrial sector is exceptionally unique as for procedure, work, and hardware the executives, IoTempowered ongoing Judgment Support Systems are fundamentally significant frequently through procedure mining and streamlining strategy [7].

\section{Contribution of Cloud Computing and Machine Learning in the Integration of Smart City Technology}

According to the Cisco report, the number of objects connected to the Internet has exceeded the number of human beings in the world. Reports show that the number of Internetconnected devices is expected to increase twofold from 22.9 billion in 2016 to 50 billion by 2020 as shown in Figure 3 . Most IoT applications do not only focus on monitoring discrete events but also on mining the information collected by IoT objects. Most data collection tools in the IoT environment are sensor-fitted devices that require custom protocols, such as message queue telemetry transport (MQTT) and data distribution service (DDS). Given that sensors are used in nearly all industries, the IoT is expected to produce a huge amount of data. The data generated from IoT devices can be used in finding potential research trends and investigating the impact of certain events or decisions [8]. These data are processed using various analytic tools. Figure 4 illustrates the process of data collection, monitoring, and data analytics. Although IoT has created unprecedented opportunities that can help increase revenue, reduce costs, and ameliorate efficiencies, collecting a huge amount of data alone is insufficient. To generate benefits from IoT, enterprises must create a platform where they can collect, manage, and analyze a massive volume of sensor data in a scalable and cost-effective manner. In this context, leveraging a big data platform that can assist in consuming and reading diverse data sources as well as in accelerating the data integration process becomes vital. Data integration and analytics allow organizations to revolutionize their business process. Specifically, these enterprises can use data analytics tools to transform a huge volume of sensor-collected data into valuable insights [9].

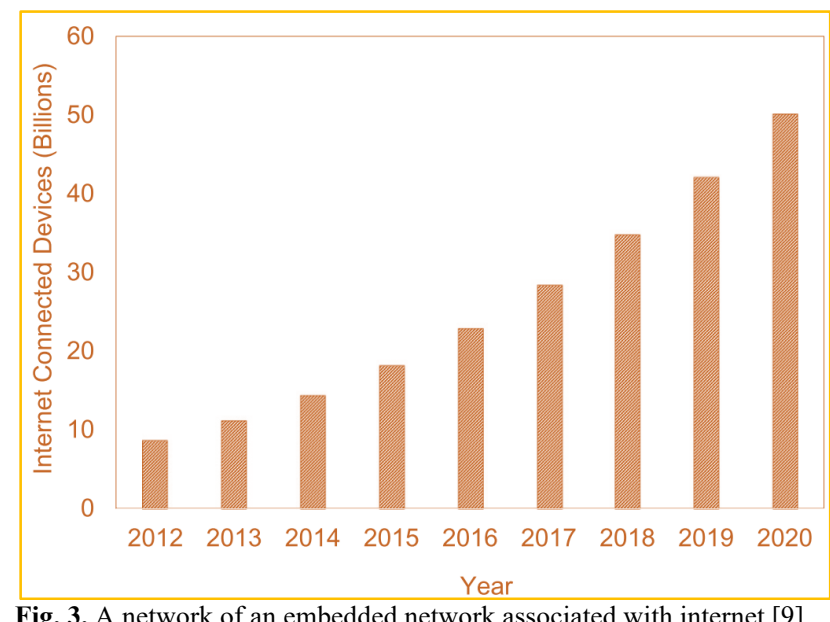

Fig. 3. A network of an embedded network associated with internet [9]

Since IoT will be among the most significant sources of new data, data science will provide a considerable contribution to making IoT applications more intelligent. Data science is the combination of different scientific fields that uses data mining, machine learning, and other techniques to find patterns and new insights from data. These techniques include a broad range of algorithms applicable in different domains [10]. The process of applying data analytics methods to particular areas involves defining data types such as volume, variety, and velocity; data models such as neural 
networks, classification, and clustering methods, and applying efficient algorithms that match with the data characteristics [11]. A detailed literary analysis of recent researches concerned with IoT based big data and analytics is summarized in Table 1.

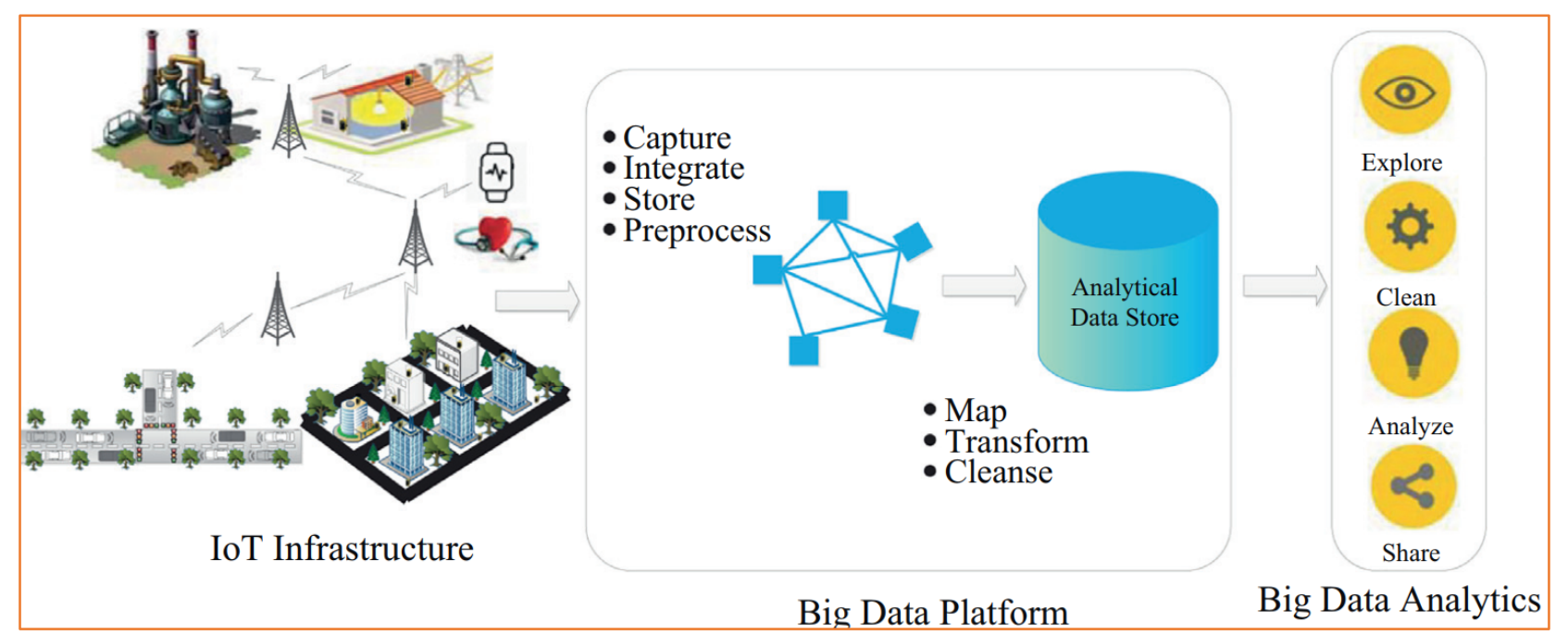

Fig. 4. Intercorrelation of IoT, Big data and machine learning [10]

Table 1. Literature analysis of the recent contribution of IoT in various sectors with analytical techniques

\begin{tabular}{c|c|}
\hline Authors & $\begin{array}{c}\text { Proposed theme } \\
\text { with IoT }\end{array}$ \\
\hline $\begin{array}{c}\text { Bashir and Grill } \\
\text { Lee et al. [13] }\end{array}$ & $\begin{array}{c}\text { IoT big data } \\
\text { analytics framework } \\
\text { IoT-based cyber- } \\
\text { physical system }\end{array}$ \\
$\begin{array}{c}\text { Rizwan et al. } \\
\text { [14] }\end{array}$ & $\begin{array}{c}\text { IoT based traffic } \\
\text { management system } \\
\text { Firework, a new } \\
\text { computing paradigm }\end{array}$ \\
\hline
\end{tabular}

Rathore et al. [16]

Ahlgren et al. $[17]$

Sezer et al. [18]

Cheng et al. [19]

Wang et al. [20]

\begin{tabular}{|c|}
\hline Key elements \\
Oxygen level, \\
smoke/hazardous gases, \\
and luminosity \\
Information analysis \\
and knowledge \\
acquisition methods \\
to improve productivity
\end{tabular}

Low-cost traffic detection sensors Merge multiple data views into a single job to perform detailed data analytics

Smart city management system

Green-IoT platform

Augmented framework-semantic web technologies, big data, and IoT Geelytics, an edge analytics platform

IoT and big data for the maritime cluster

Weather and

water sensors, vehicular networking sensors, surveillance objects,

smart home sensors,

and smart parking sensors

Transportation, air quality, and energy efficiency

Five layers of architecture

System characteristics of heterogeneous edge/cloud nodes, and the current system workload

A new framework for integrating industrial IoT with big data

Analysis of interpretation
Specifically designed for smart
buildings towards smart cities
and smart airplanes
New context intelligence
framework-
industrial informatics based on
the sensors, locations, and
unstructured data for big data
mining

To analyze traffic density-

better than existing technology

Firework aims to minimize data access latency

Data generation, data

Computing platform

Cloudera Hadoop

distribution using pyspark

Industrial big data analytics

Predictive analytics

Distributed data processing and sharing

in an IoT-based,

collaborative edge environment

Mapreduce Hadoop ecosystem

gathering, data combining, data categorization, data preprocessing,

and decision-making using Spark over Hadoop

IoT to deliver

services for based on open data and standards, including interfaces and protocols Data acquisition, extracttransform-load (ETL), semantic-rule reasoning, learning, and action Support dynamic stream processing

Increase output and productivity in the global maritime industry
Open platforms and open data for the development of smart cities

Machine-learningbased models

Geo-distributed analytics

Parallel processing with Mapreduce and low-latency 


\begin{tabular}{|c|c|c|c|c|}
\hline & & $\begin{array}{l}\text { and analytics } \\
\text { technologies }\end{array}$ & & \\
\hline $\begin{array}{c}\text { Prez and Carrera } \\
{[21]}\end{array}$ & $\begin{array}{l}\text { Multi-tenant } \\
\text { data stream } \\
\text { processing }\end{array}$ & $\begin{array}{c}\text { Performance } \\
\text { characterization of the } \\
\text { servIoTicy API }\end{array}$ & $\begin{array}{c}\text { Advanced querying } \\
\text { mechanisms, multi-protocol } \\
\text { support for hosting workloads } \\
\text { in the cloud }\end{array}$ & $\begin{array}{c}\text { Advanced data-centric } \\
\text { technologies }\end{array}$ \\
\hline Jara et al. [22] & $\begin{array}{c}\text { Bid data with cyber- } \\
\text { physical } \\
\text { systems }\end{array}$ & $\begin{array}{l}\text { Cloud security and the } \\
\text { heterogeneous } \\
\text { integration of data }\end{array}$ & $\begin{array}{l}\text { Real-time stream data } \\
\text { processing with client key } \\
\text { architecture }\end{array}$ & $\begin{array}{l}\text { Data discovery } \\
\text { mechanisms }\end{array}$ \\
\hline Ding et al. [23] & $\begin{array}{l}\text { IoT paradigm } \\
\text { (IoTstatisticdb) }\end{array}$ & $\begin{array}{c}\text { General statistical } \\
\text { database cluster } \\
\text { mechanism for big data } \\
\text { analysis }\end{array}$ & $\begin{array}{l}\text { Distributed } \\
\text { and parallel fashion using } \\
\text { multiple servers }\end{array}$ & $\begin{array}{c}\text { Database management } \\
\text { systems } \\
\text { (DBMS) kernel }\end{array}$ \\
\hline $\begin{array}{l}\text { Vuppalapati et } \\
\text { al. [24] }\end{array}$ & $\begin{array}{l}\text { Big data in } \\
\text { healthcare }\end{array}$ & $\begin{array}{l}\text { Integrating massive } \\
\text { data points with } \\
\text { electronic } \\
\text { health records (EHR) }\end{array}$ & $\begin{array}{l}\text { Scalable cloud architecture for } \\
\text { real-time monitoring of health } \\
\text { status }\end{array}$ & $\begin{array}{c}\text { Apache } \\
\text { Kafka and Spark }\end{array}$ \\
\hline $\begin{array}{l}\text { Mukherjee et al. } \\
{[25]}\end{array}$ & $\begin{array}{l}\text { Condor, a grid } \\
\text { framework }\end{array}$ & $\begin{array}{l}\text { Data-parallel execution } \\
\text { in the IoT paradigm }\end{array}$ & $\begin{array}{c}\text { Condor has better scalability } \\
\text { and } \\
\text { CPU utilization for data- } \\
\text { parallel iobs }\end{array}$ & $\begin{array}{c}\text { Server-based } \\
\text { architecture with three- } \\
\text { tier }\end{array}$ \\
\hline $\begin{array}{l}\text { Berlian et al. } \\
\text { [26] }\end{array}$ & $\begin{array}{l}\text { Internet of } \\
\text { Underwater Things } \\
\text { (IOUT) framework }\end{array}$ & $\begin{array}{l}\text { Testing the } \\
\text { applicability of the } \\
\text { scalable } \\
\text { trust management } \\
\text { protocol with IOUT }\end{array}$ & $\begin{array}{l}\text { Trust-based admission control } \\
\text { for IOUT systems }\end{array}$ & Mapreduce \\
\hline
\end{tabular}

Often ICT (Information \&Communication Technology) tools for a smart city deal with different application domains such as land use, transport and energy, and rarely provide an integrated information perspective to deal with sustainability and socio-economic growth of the city. Smart cities can benefit from such information using big, and often real-time, cross-thematic data collection, processing, integration, and sharing through inter-operable services deployed in a cloud environment [27]. However, such information utilization requires appropriate software tools, services, and technologies to collect, store, analyze and visualize large amounts of data from the city environment, citizens, and various departments and agencies at the city scale to generate new knowledge and support decision making.

\section{Industrial Sector Progressive Development by IoT \&AI}

In the industrial application automation with a higher perceptual layer of the Internet of Things is involved due to them because of the vision of worldwide foundation interconnectivity. To build up an enhanced industrial IoT platform, various advancements must be incorporated. The quality of service and security gave by various advancements is another challenge for standard industrial norms. In considerations like dependable, adaptable, versatile, and issue tolerant systems must not be undermined to accomplish the target of an automated industry [28]. So also, exceptionally adaptable information stockpiling and preparing stages that are bolstered by productive cloud-based administration choice is an open challenge. The IoT application quality rate gave by these advances must be good before a keen industrial application is completely incorporated. Computational insight automation and controls are successful, productive, and vigorous in information designing, which involves delicate figuring, AI, and information mining. In any case, the adequacy, productivity, and vigor of computational intelligence are constrained to little informational collections [29]. The huge information produced from the monitoring industrial fields has rendered the current computational knowledge calculations insignificant in enormous information examination. At the point when the limit of the informational collection expands, the viability, productivity, and heartiness of the computational knowledge calculations regularly lessen, in this manner making them unseemly for investigating information in huge information produced from the database industrial management [30]. When this scenario exists, big data analytics comes into the role to support the smart technology and smart embedded sensors with high-end data processing and communicating techniques. Figure 5 shows an overview of the Industrial IoT framework with other advanced technology [31].

\section{Configurations of IoT Layer}

IoT serves as a potential solution to drive essential processes of the many industrial zones more efficiently and reliably [32]. An existing IoT architecture for remote monitoring and control comprises of five-layer has been fashioned to three layers progressing to accumulate all the requirements with the upgradation features concerning an application is detailed in Figure 6. A sensor level is invigorated with countless sensors like pressure, flow rate, temperature, vibrations, etc. and actuators like pumps, motors, and control valves, etc. The transmission level will be initiated to collect data and react to specific conditions by using either wired or wireless communication [33]. The wired technologies involved in the oil pipeline system include twisted pair wires, Digital Subscriber Line (DSL), Public Switched Telephone Network (PSTN), and fiber optic cables to transfer analog data and utilizing Ethernet, Integrated Services Digital Network to transmit digital data [34]. This communication medium establishes the link with nearby itinerant units or maintenance recruits by utilizing communication by short-range for revamping to perform conceivable actions. The wireless technology involved in the present oil pipeline communication system includes ZigBee, Wi-Fi, Mobile Radios, integrated Dispatch Enhanced Network (iDEN), Code Division Multiple Access (CDMA), Time Division Multiple Access (TDMA) [35]. In wired communication, the 
major drawback is that the requirement of the hub or network switch cards causes slower data exchange resulting in high installation and maintenance costs with a lack of mobility. The present implemented wireless technology faces the consequences like lagging in secured communication establishment, unable to enhance message integrity and throughput during communication, interference problem and poor encryption technique result in false alarm due to misinterpretation on received data with more error data [36].

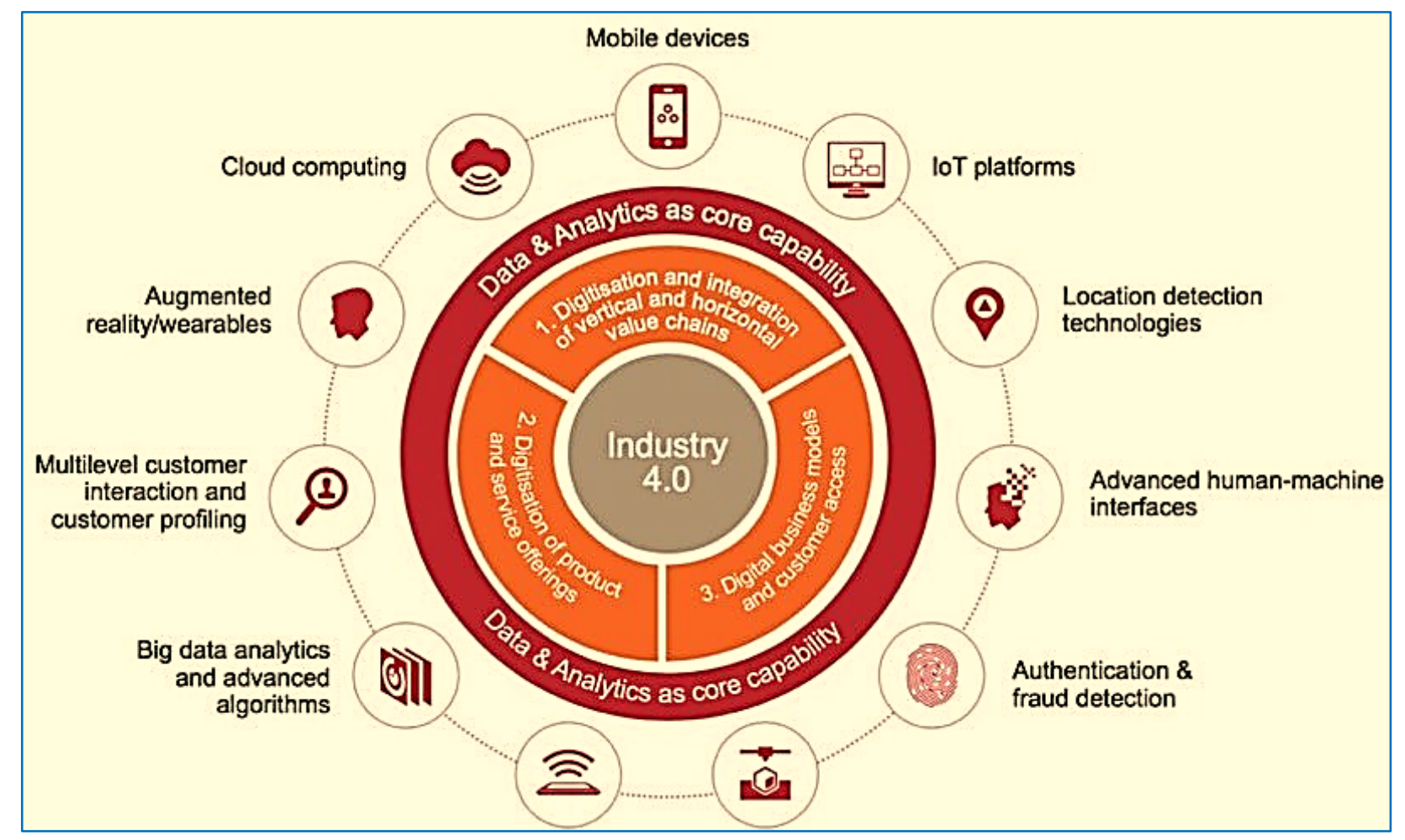

Fig. 5. Industrial IoT frame structure [31]

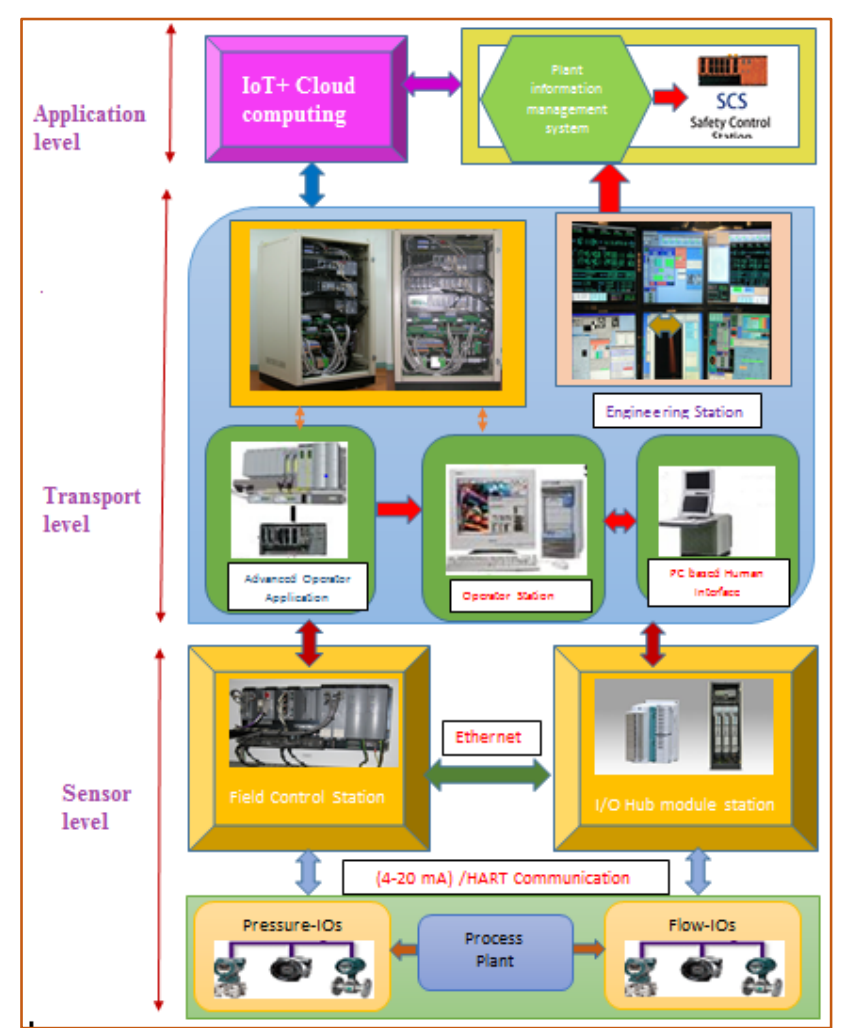

Fig 6. Each layer characterization of Integrated IoT architecture [36]

The application layer is responsible for process management like configuring a centralized database, object interfaces, service APIs, visualization tools, etc. Thus, the data gathered from the desired system is delivered to the control server through the application layer [37]. In the existing industrial sectors, the existing application layer is associated only with SCADA (Supervisory Control \& Data Acquisition) for acquiring the sensors data and makes appropriate decisions at the specified field control station but not concerned with the entire data management and processing system [38].

\subsection{Main constituents of IoT and existing protocol standards}

The main constituents of IoT involve sensors incorporated with actuators and wireless communication. It includes communication in three ways such as Infrastructure as a Service (IaaS), Platform as a Service (PaaS), and Software as a Service (SaaS) [39]. The data from the industrial application sector are stored in three categories of clouds as public, private, and hybrid. Between the cloud and the monitoring real world. Gateway plays a dominant role in establishing links to send and receive physical data. The data are transferred through different protocol standards by adapting to different magnitude and frequency rates [40]. Figure 7 gives the main essential components of IoT and Figure 8 visualizes the accompanying protocols for data communication between hardware and cloud.

The time of wave has transferred the technology from Radio Frequency Identification (RFID) and wired sensor network to the wireless by internet domain. In the IoT all the required sensor structures, data correspondence, routing addresses are oriented in one structure with various priorities. The outcome propagates in a well-measured manner by advancing a new response in imperceptibly manner [41]. The 
model-based pro-activeness is achieved in IoT by taking conventional commodities to the next level of data communications to achieve safety and productivity. The data availability limit has been crossed over, hence cloud computing comes to a major role in the industrial sector. It has been portioned IoT as two main fields as consumer and business, whereas business side orientation and growth has been higher due to high utilization and dependence [42]. It sets existing running processes as the least layer with the development of cloud techniques to access the industry administration in a powerful path. Due to the progression of Wi-Fi and 4G-LTE (Long Time Evolution) also 5G has taken a major role in data corresponding to the big data technique [43]. Nonetheless, the Internet of Things motive relies on making versatile processing and control through a portable vision-based model rather than the step-wise execution on monitoring to share the knowledge of the industrial environment in the business zones [44]. The sustaining rate and throughput rate with the IoT are meaning more than the conventional knowledge processing with the sensor system. Figure 9 shows the expectation and utility rate of IoT in the industrial sector rather than its pavement to other sectors.

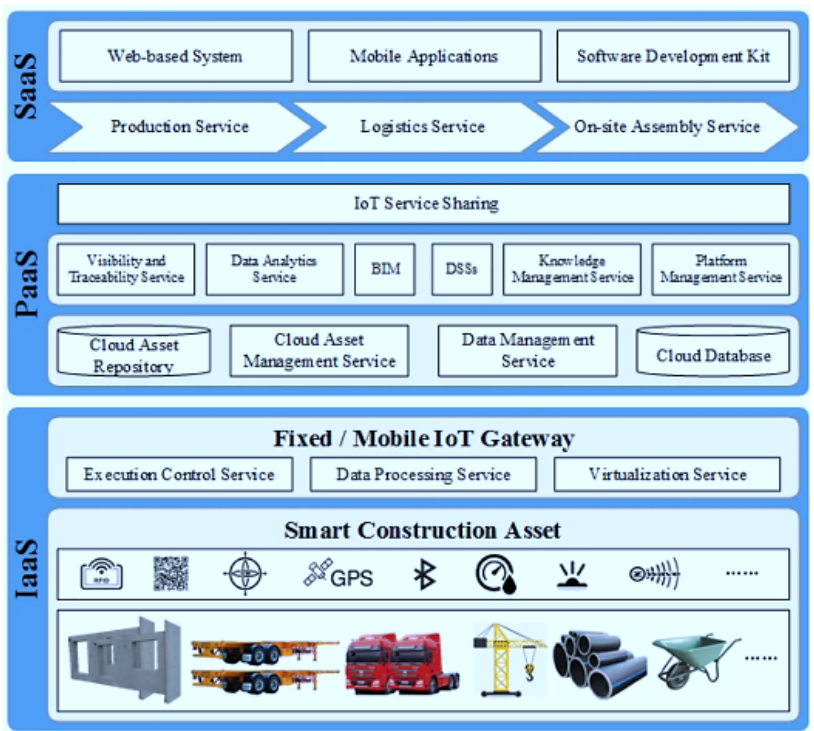

Fig. 7. Cloud-based IoT category frame line [40]

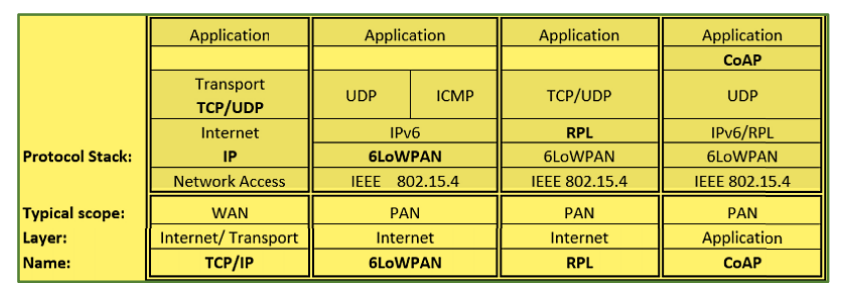

Fig. 8. IoT protocol supporting structure [41]

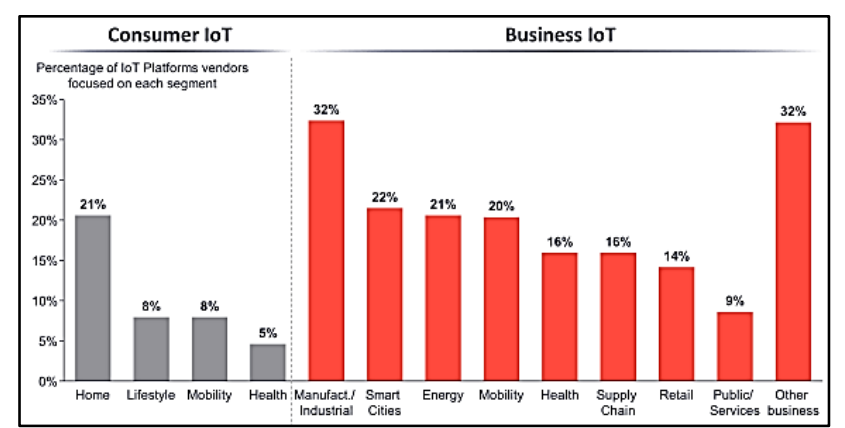

Fig. 9. Statistics of IoT contribution in Industries and manufacturing sectors in comparison with other sectors [44]
In recent years, communication occurs wirelessly to the remote information analytics center to examine and interpret the process behavior and afford the appropriate decision in case of catastrophic circumstances [45]. An automated control frame structure in a tedious transportation system still relies on the performance of the Proportional-IntegralDerivative (PID) controller to assure safety in a commercial manner [46]. This is accomplished by continuous monitoring of desired field parameters such as viscosity, flow rate, temperature, velocity and pressure to make a prompt decision like changing the stem position in the valve, changing the speed or frequency of the pump so that system field parameter retains on its threshold range. The main stimulus for process control technology is safety since the loops having adequate performance are only $68 \%$ which are in manual mode. Hence in recent scenarios, the need for an advanced controller is increased to determine the optimal system performances [47].

SCADA systems used in process plants are not scalable due to slow data processing with minimum memory storage and non-feasible when the software or protocol upgradation availability resulting in high maintenance cost [48]. Hence, the Industrial Internet of Things is investigated towards the development of inspection techniques to detect the risk occurrences incorporated with the hardware system. In this context, all modernized industries have been undertaken the IoT platform synchronizing communication between the hardware elements (sensors, actuators, control panel, etc.) and networked embedded data devices [49]. This frame structure collects appropriate field data which will be conveyed to the remote supervision center for analysis and processing to afford a suitable rapid decision in case of catastrophic situations. Ubiquitous sensing enabled for monitoring and control in the industrial sector are mostly WSN (Wireless Sensor Network) system is still incompatible because the quick-change over of industrial constraints are not interconnected [50]. Nowadays, SCADA systems are not climbable due to derisory memory and processing time, offers high cost of hardware drivers and management, not malleable when a process plant hardware needs to be updated, unyielding due to the data orientation-based protocol variation and huge data logging results in delay-based output model rate [51]. Hence, the Human interfaced Internet of Things and the Engineering Internet are reconnoitered towards the improvement of modules used to identify the manifestation of impairment. In this upgradation era, all renovated industries have been assured using the emerging methodology IoT which permit a ubiquitous bonding between the process plant hardware (sensors, actuators, control panel, etc.), smart hybrid objects and networked implanted devices to accumulate applicable data that will be communicated wireless manner [52]. In the remote information analytics center, acquired data has to be examined and modulated to construe the process and afford the appropriate decision in case of calamitous circumstances [53].

\section{IoT Applications in Major Sectors}

In most of the industrial sector, the success of the Internet of Things was achieved with the modernized growth of miniaturized microdevices fabrications. It headways the main merits of dwindled space exploration with the same features and characterizations. Due to the micro-computerized hardware, remote communication is made feasible and ecologically comfortable to convey the control processes 
[54]. The distributed compact remote system has taken the IoT platform as the core to sustain and envision the situation handling in the industry with various parameter estimations. The improvement from very-large to the small with software structured remote sensor and communication system is providing proficient detection and versatile understanding of industrial knowledge[55]. In basic, total IoT has a better capacity of knowledge source, and sharing with prompt control and decision plays a predominant role with forecasting ability. The resources with structures protocol are followed to envisage the perceived quality of the market to allow IoT to put forth in the top layer with the ultimate acknowledgment register. Virtualized capacity innovations are the distributed algorithm coded computing to make the data server strong and progressive. This stage goes with furnishing the client with straightforward electronic representation as it modes to model calibration. The omnipresent structure for detection and estimation, to sort out the client sights on the quality and quantity based model development with things connected wirelessly utilizing the internet to respond when the reactiveness gets more demands. As we move from www to web2, the obligation for statistics with programmed facts on-request based natural inquiries increments pathways establishes quicker accessibility output [56]. To exploit the accessible web revolution requires firewalls to send enormous scale, commandable independent, remote executives, and preparing invitation, and investigation. Cloud figuring affords extreme loss rate recovery scheme with server management with dada statistics with dynamic revelation, adaptability, and composability to make the standards for cutting superiority Internet of Things applications [57].

\subsection{Role of IoT in smart city application}

As urban exhibitions depend on the physical framework as well as on the accessibility, nature of information correspondence, and social foundation. The key empowering agent of these smart city applications is perhaps the IoT where regular articles and gadgets are associated with the system advancements. The guarantee of a keen city prompts an exponential increment in information by a few sets of greatness [58]. Thusly, such tremendous volumes of information or huge information are at the center of the administrations rendered by the IoT. The marvel of enormous information has for quite some time been portrayed by volume, speed, and an assortment of information types that have been made at a consistently expanding rate [59]. Huge information offers the potential for the city to acquire significant experiences through acquisition from continuous data logging with powerful firewalls and gateway systems. Through the versatility modes of resource afford unstructured data that should be processed with programmed estimator on the dynamic algorithm eventual rate [60].

Figure 10 outlines the scene of the keen innovations with enormous information and distributed computing, in which different savvy applications trade based data correspondence. Each received unstructured and structured data are segregated with each token pass in the cloud server [61]. The circulating rate is fixed with each unstructured format and it is finalized with the required sensitivity to generate structured command. The processing and analysis are done in the same phase with the frame rate of high and low alternatively to balance the data field of the handshaking path of utilization criteria [62]. For example, not only SQL, which is used to improve a solitary help or application and is shared among different administrations. Hence, the programming model for preparing huge informational collections with parallel calculations can be utilized for information investigation to get an incentive from the put-away information [63]. Brilliant urban areas have assumed a key job in changing extraordinary regions of human life, contacting segments, for example, transportation, wellbeing, vitality, and instruction. For instance, the information on climate data is fundamentally expanding at a quick pace. Distinguishing and acquiring important data from a lot of climate information can be incredibly helpful as far as a horticultural improvement [64]. Besides, the examination of the climate information can help advise the individuals ahead of time about the conceivable dangerous conditions.

The framework structure of enormous information in the keen city, as appeared in Figure 10, can be isolated into numerous layers to empower the improvement of coordinated enormous information the executives and IOT incorporated city advancements. Each layer speaks to the potential usefulness of huge information keen city segments [65]. The principal layer is the arrangement of articles and gadgets associated with employing neighborhood or potentially wide-region systems [66]. In the subsequent layer, all the gathered unstructured information are put away in a common disseminated flaw tolerant databases found either in the city server farm outfitted with all system components or by enormous information stockpiling, for example, S3, Google cloud administrations, and Sky blue from merchants, for example, Amazon, Google, Microsoft, and Cloudera [60]. Different huge information store frameworks can be utilized in these cases such as Cassandra, Hbase, MangDB, CouchDB, Voldemort, DynamoDB, and Redis. Inside a similar layer, the put-away information is prepared to rely upon the approaching questions utilizing a clump-based programming model, for example, MapReduce system or other preparing motors utilized for huge information [67]. MapReduce gives an amazing programming model to parallel and conveyed the handling of huge information on groups. In-stream handling, information must be prepared rapidly to onsite the monitoring rate to change progressively to balance the extreme modifier schedule to achieve in a brilliant city condition. Numerous innovations can help procedure and follow up on ongoing spilling unstructured information continuously, for example, Spark, Storm, and S4 [68].

\subsection{Role of IoT in the construction sector}

Notwithstanding, practically speaking, issues still exist that hamper the presentation of pre-assembled development. Right off the bat, as per our examinations, the degree of reception of Information Technology (IT) is low in most preassembled development ventures [69]. Customary techniques still rule information accumulation and transmission, which are mistaken, wasteful, deficient, and blunder inclined. Such inadequacies prevent opportune reactions when changes or interferences happen. Besides, data is dispersed and compartmentalized among different partners, for example, the makers of pre-assembled segments (or prefab producers), coordinations organizations, temporary workers, furthermore, clients [70]. This obstructs successful coordination and collaboration among them and may prompt erroneous stock, delayed lead time, late conveyance, and lost prefabs. It has just been recognized as a significant hindrance to productivity and could demonstrate a countervailing power against the upsides of pre-assembled development. Web of Things (IoT) 
and distributed computing give openings to take care of these issues and have demonstrated powerful in assorted fields, such as crisis the board, producing frameworks, and the petrochemical business [71]. Figure 11 shows the IoT platform architecture for the construction sector with specified requirement levels.

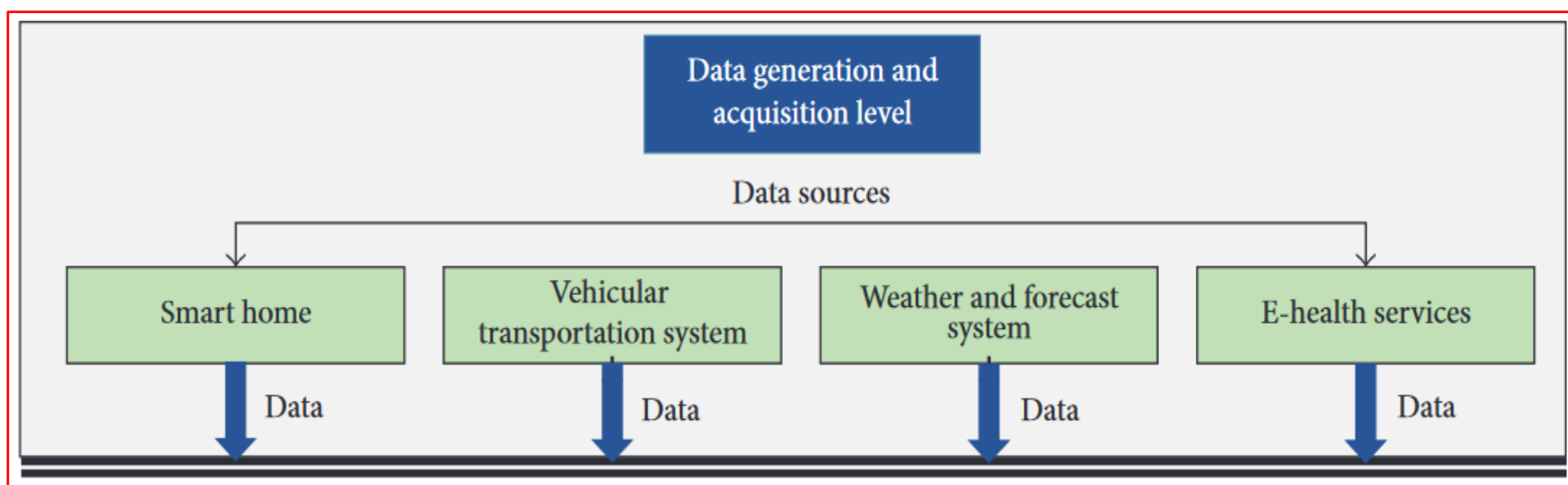

Communication technologies

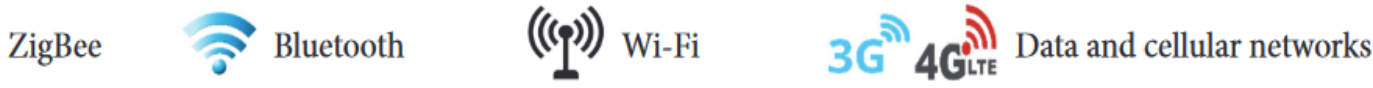

Data management

and processing level

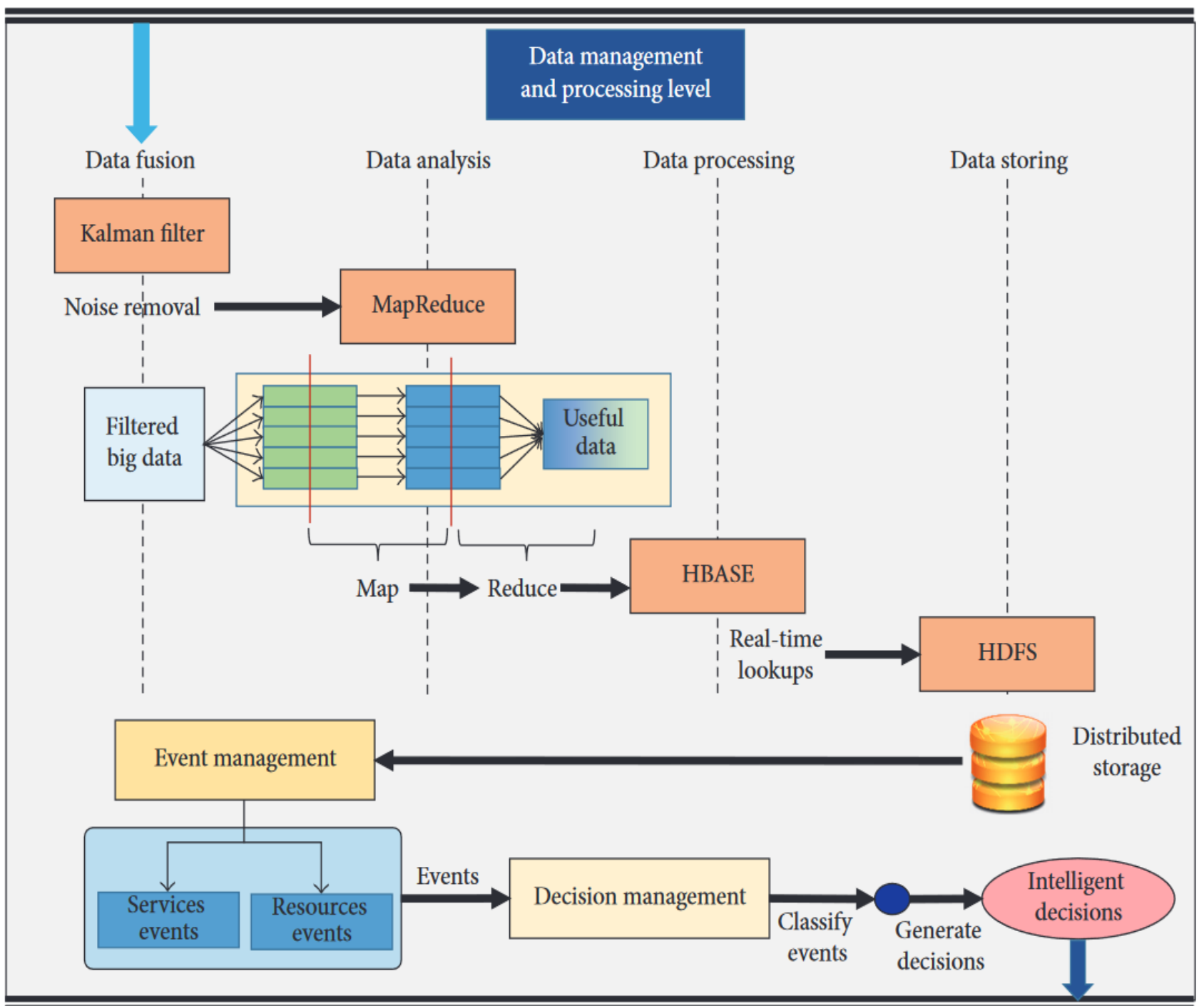

Fig. 10. IoT in smart city with Big data computation technology using MapReduce [64]

All the more explicitly, in the development business, different endeavors have been attempted, extending from vitality the executives, security the board, to materials the board [72]. Be that as it may, most past examinations have concentrated on either explicit period of development ventures or the conventional cast-in situation development, which can't be straightforwardly applied to the entire procedure of pre-assembled development. The premier point is that prefabs are one of a kind and basic [73]. They require thing level ID and information gathering to acknowledge finegrained the board all through the entire prefab inventory network, while in customary development, materials are 
fundamentally overseen by the clump. Moreover, to limit lead time, wipe out approaching inadequacies, and improve versatility under interferences, it requires a consistent joint effort among every one of the partners included [74]. Then, different explicitly planned advancements accessible for construction should be well-incorporated to completely figure it out their advantages [75].

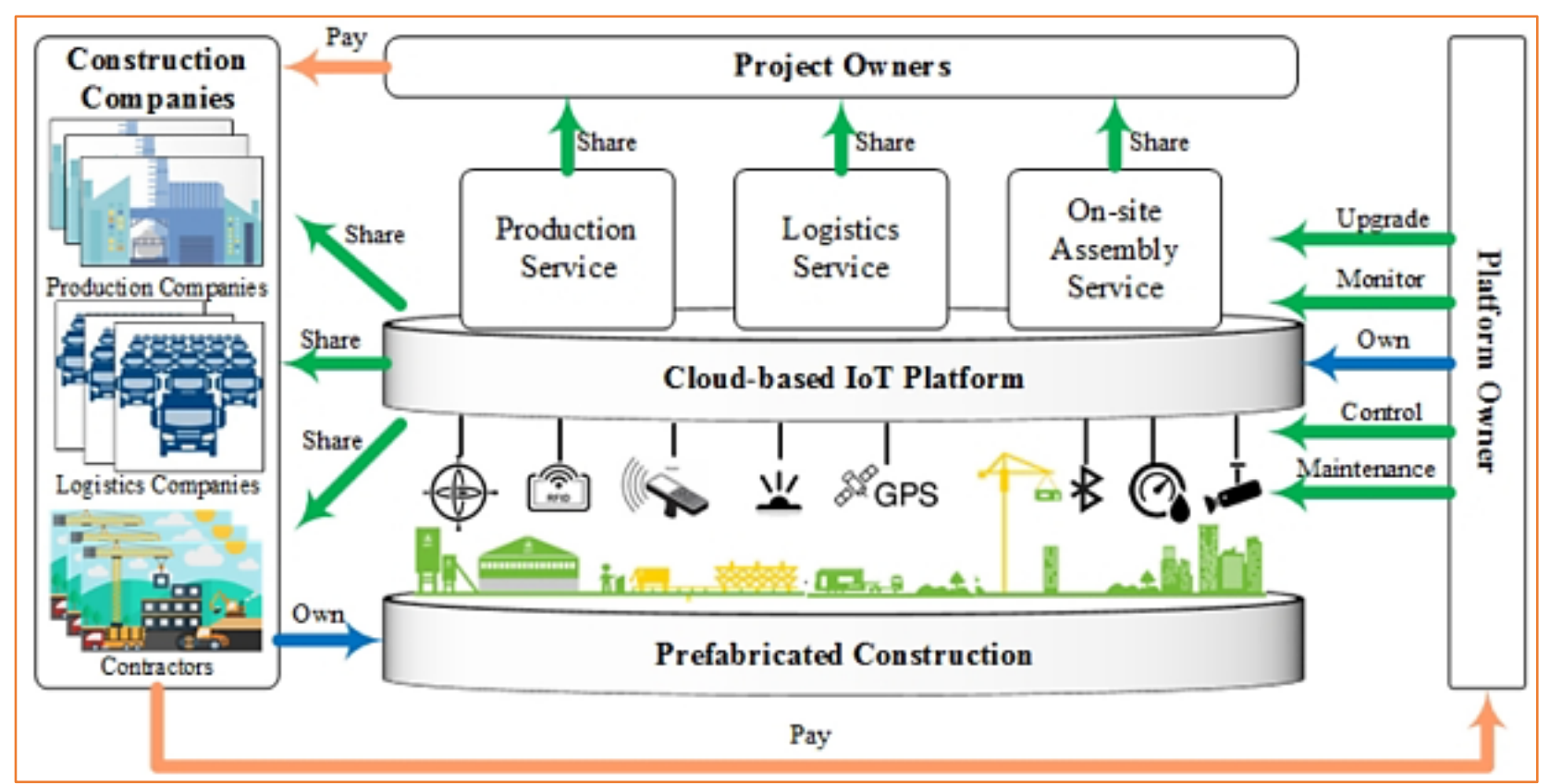

Fig. 11. IoT platform with specified levels of construction sectors [71]

Against this foundation, challenges still exist that block the selection of these advancements in pre-assembled development. Initially, most organizations engaged with preassembled development are little and medium enterprises, which will be unable to manage the usage of IoT frameworks [76]. From one perspective, executing IoT frameworks requires high introductory venture, and ceaseless upkeep costs a short time later, which are trying for Medium enterprises [77]. On the other hand, pre-assembled development undertakings vary from one another and typically have various necessities on IoT frameworks. Because of the noteworthy advancement works and time utilization, it is outlandish for the construction sector to manufacture explicit frameworks to adapt to the differing preassembled development extends that they took an interest in during various periods [78].

\subsection{Role of IoT in the data mining sector}

Prognostics is the expectation of the wellbeing status of observed gear. There are three kinds of approaches for prognostics: model-based, master framework, and information-driven. The model-based methodology is hard to execute for complex frameworks [79]. It endeavors to utilize scientific models, which are profoundly coupled to the hardware and area, to speak to the conduct of the gear. The master frameworks approach requires specialists' learning to assemble the framework. Such frameworks are worked by characterizing disappointment modes and distinguishing corruption designs of the hardware [80]. Such a framework is additionally firmly coupled to the gear and space. The information-driven methodology for the most part utilizes present and chronicled sensor information and functions admirably with complex frameworks [81]. Such a framework is increasingly relevant to numerous spaces since it is simply information-driven and isn't coupled to explicit hardware.
Prescient upkeep brings down costs by avoiding disappointments, unscheduled upkeep, and personal time, and by guaranteeing the substitution of coming up short parts is done just when required. To bring down these expenses dependably, sensor choice must be directed with care [82]. Figure 12 represents the outline of the IoT frame for data mining when the main control hub is accounted for with IoT characterization. Master information of basic segments, information-driven entropy-based methodologies, or a mix of the two strategies are instances of sensor choice systems. It is partitioned into three layers (the observation layer, the middleware layer, and the application layer), which are kinds of the IoT layers referenced in segments [83]. The observation layer abstracts the mist and implanted frameworks. It performs detecting, lightweight stockpiling, systems administration, and AI. It gives the interface to low-level hubs. The middleware layer abstracts the haze and the cloud and for the most part performs all the more substantial stockpiling, systems administration, and AI contrasted with the observation layer. It gives the interface to recognition layer hubs [84]. The application layer is like the IoT application layer referenced in segment 2. Found in the observation layer, the vehicle hub (VN) speaks to the vehicle. The VN has a J1939 arrange and a portal [96]. By perusing J1939 traffic, the VN's entryway can perform sensor information procurement, conglomeration, and lightweight information examination. Information is put away utilizing an installed database since it is lightweight [30]. The door interfaces the J1939 system to the armada framework. Each $\mathrm{VN}$ has a product regulatory operator introduced on the door, enabling overseers to remotely refresh the VN's product [85]. Since the $\mathrm{VN}$ is versatile, it must have arranged an association using remote advancements, for example, Wi-Fi and LTE. MQTT is utilized as the machine-to-machine convention for VN-to-armada correspondence. 


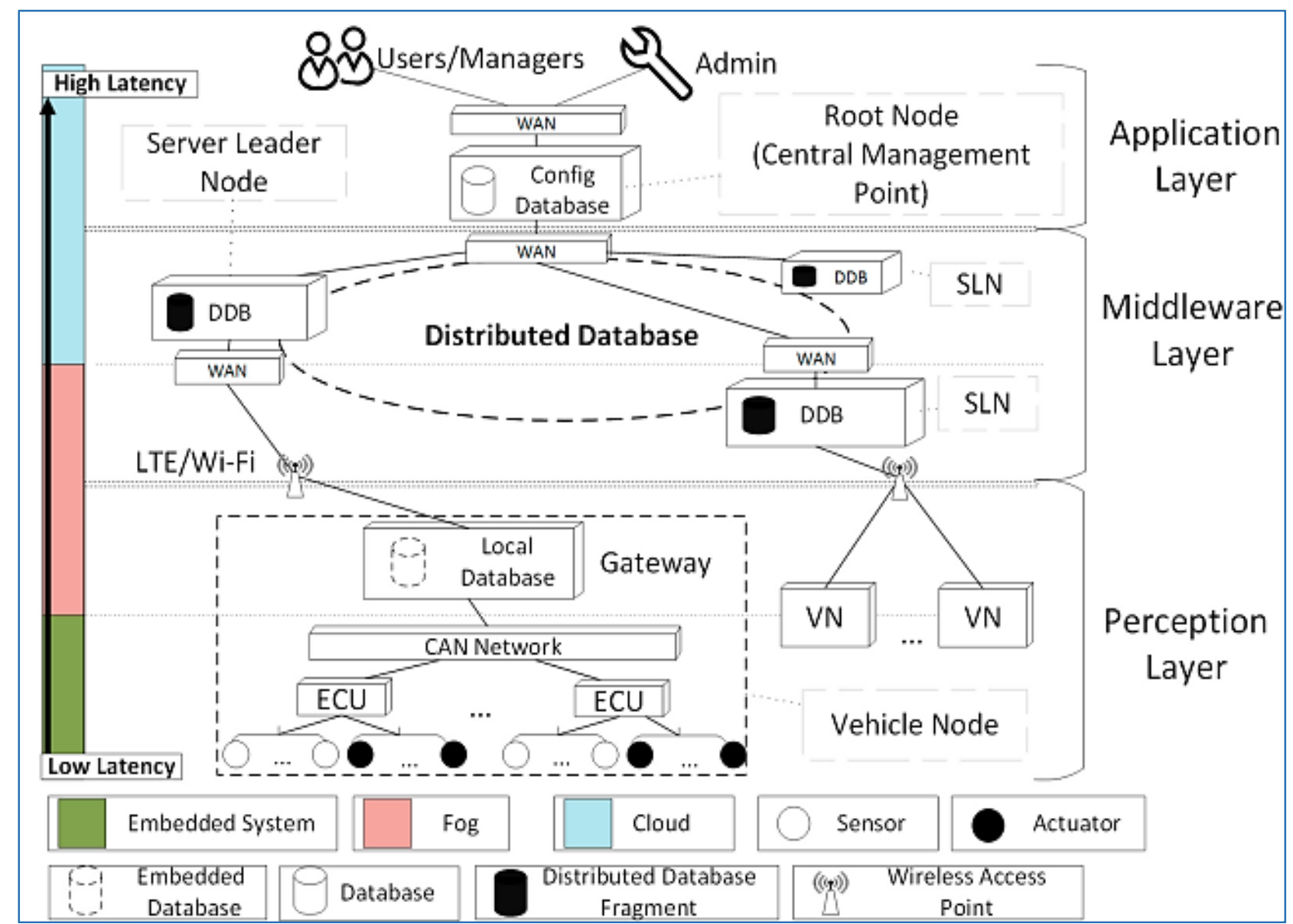

Fig. 12. IoT paradigm for the data mining sector with sensor node protocol specifications [81]

The server chief hub (SLN) is found in the middleware layer. It tends to be found in the haze (an asset obliged cloud) or the cloud [86]. It is answerable for overseeing VNs of a topographical district. The SLN has an MQTT dealer, which gives its VN armada the capacity to speak with one another and the SLN itself. Each SLN stores sections of the armada framework's disseminated database (DDB). A district's armada information can be put away provincially in its SLN's DDB section, which gives information territory, and assures builds accessibility [87]. By structure, the SLN can perform wide information investigation. Found in the application layer, the root hub $(\mathrm{RN})$ is liable for dealing with the whole armada framework [88]. It is the framework's focal passageway, gives the interface to IoT applications, associates armada hubs to the framework, and empowers wide organization.

\subsection{Role of IoT in the oil and gas industry}

In the oil and gas industry, the role of monitoring and control is quite important with continuous data processing. Because it includes the data acquirement from both internal and external environment because the risk occurrences were dependent on both [89]. Hence various sensors are mounted at required evaluated locations to obtain the field pipeline data efficiently. But the role of IoT application in the oil and gas industry is still in the research step of analyzing with various constraints. However, the reliable IoT frame structure will bring an imminent solution to the existing problems in the pipelines ecologically and scientifically of schemes [90]. During transportation through pipelines, the materials of oil and gas components subjected to more risk rates due to environmental factors and internal pipeline influences. Hence any risk rate gets higher, immediately there is a drop out of production rate with the major drawback to the environment and public. To sort out these types of risk rates, many sensorsoriented group structures are developed for the oil and gas industry to get instant parameter statistics to afford smooth safety enhanced product distribution. Due to this, the Internet of Things comes into the online virtualization and maintenance of pipelines for long runs with specified technology upgradation [91]. It ensures the collection of entire pipeline system data regarding sensors, fluid characterization, and finally substation control elements. The highly enumerated wireless connectivity using an internet platform a smart oil and gas transportation pipeline system is formulated. In the central supervision and analysis center, the desired control execution will be generated and distributed to each substation in an orderly manner with a prompt decisionbased control model [92]. The control supervision functions together to implement the following steps:

- Calibrating the risk rate with sensor node evaluation.

- Refreshing remote terminal with proper coding to nullify the data transmission delay.

- Processing the pipeline sensor data in a priority manner to make a decision algorithm.

- Alerting the control server with a throughput of risk rate at every specified time interval rate.

- Protocol assessment with proper sensor to sensor data communication to the actuator node.

- Cloud server data storage and data analytics with revamped flow path execution ratio based on the quantity model to generate emergency alert during the risk assessment.

- Maintenance and sensor calibration data valuation concerning the pipeline's lifetime and sensors data. 
The implementation of IoT to the oil and gas industry has performed in a better way by supporting the local control system with early warning and online diagnosis [93]. Figure 13 shows the layer-wise operating performance of IoT concerning the oil and gas industries. Each layer characterization depends on the pipeline and field parameters safer limit rates. Based on the computation algorithm local server performs control action within the substation. When the impact has been progressed, the risk rate gets increased, the IoT architecture proceeds in an intelligent way to resolve the situation by minimizing the impacts in a smarter online portable way [94].

The IoT role in an oil and gas pipeline system is to take over the entire pipeline system irrespective of distance and locations with enhanced sensors and actuators association through secured data transmission to the cloud [95]. An IoT in the oil and gas industry can sort out the following issues: leakages or breaks-up, voids, corrosion rates, oil thefts, depositions in the pipelines, etc [96].

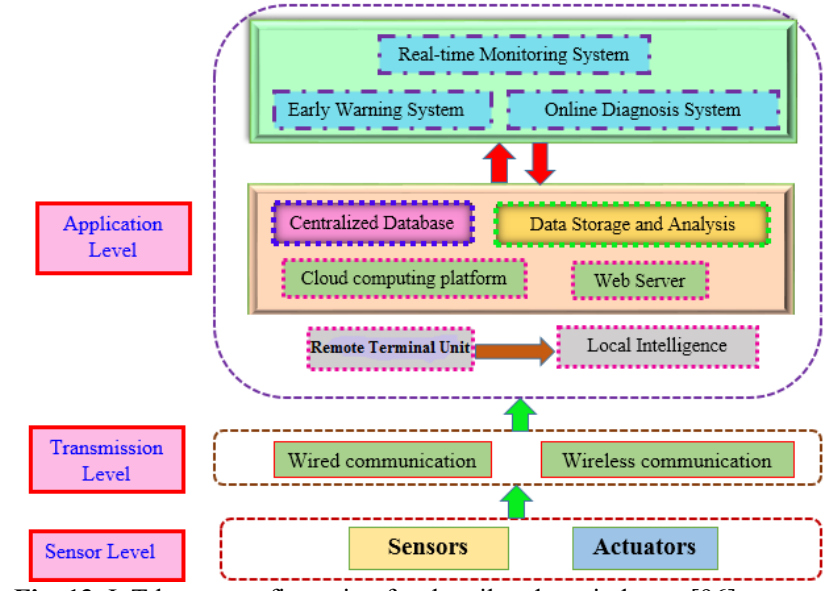

Fig. 13. IoT layers configuration for the oil and gas industry [96]

\subsection{Role of IoT in Healthcare}

Existing examination points Body-to-Body Network (BBN) as a work organization that utilizations individuals to transmit information inside a restricted geographic region. This idea can promptly manage client portability necessity too as issues like vitality productivity and unwavering quality of service (QoS). Such multi-jump correspondence offers the likelihood to interface bigger sensor organizes by empowering progressively remote connections between every hub [97]. The WBANs are associated with one another and information directing is made to move the information up until the connector gadget (i.e., the entryway). In this manner, BBN configuration ought to consider a vitality proficient steering, QoS-mindful traffic the board, and versatility of the executives. Nonetheless, new and customary difficulties show up with the BBN idea for example, remote channel engendering issues (vitality proficiency, impedance, and conjunction) of various correspondence advancements that offer a similar radio range, interoperability issues, moral difficulties [98]. Another arrangement that has been prescribed to improve IoT arrangements in human services is parallel preparation.

Transmit level parallelism (TLP) comprises of handling diverse wellbeing information streams in parallel. The utilization of multicore innovation empowers the streamlining of accessible assets regarding preparing execution and power utilization. Therefore, the sensor organizer can have an expanded lifetime. A low reception rate could be overseen by giving an IoT arrangement a worth driven methodology rather than an innovation-driven one. Along these lines, the marketable strategy is as significant as the integrative engineering of the IoT arrangement [99]. To incorporate inhome social insurance in a bigger IoT arrangement, proposed a field-tested strategy that comprises of a helpful environment between conventional medicinal services suppliers and Internet content suppliers. Besides, various sorts of ITempowered abilities could shape, and be formed by, the home human services supplier's reactions to natural changes. Figure 14 shows the architecture network based on body sensor mesh hub with IoT implementation in healthcare industrial research.

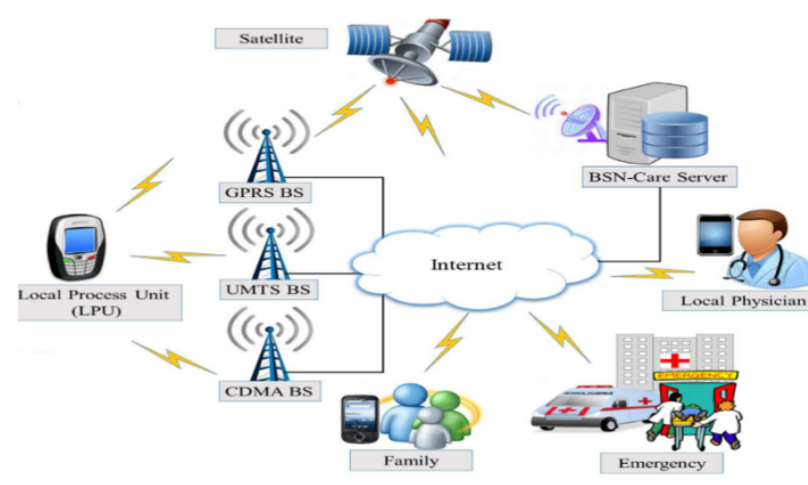

Fig. 14. IoT application architecture in healthcare applications [99]

Thus, preparing and mindfulness battles have a significant job in more extensive reception likewise prescribes adopting a piecemeal strategy to bring IoT advancement in medicinal services to maintain a strategic distance from the flexibility from this specific traditionalist industry [100].

\subsection{Role of IoT in Education}

Instruction has transformed from an information move model to a functioning communitarian self-coordinated model by the troublesome impact of innovation in the present instructive foundations. This has constrained numerous foundations to reevaluate instructing and learning [101].

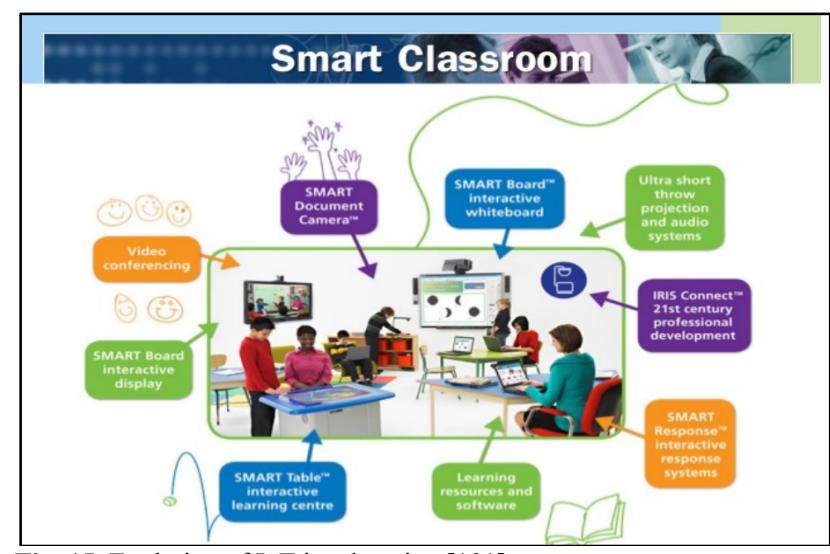

Fig. 15. Evolution of IoT in education [101]

The impact of innovation can be seen in numerous parts of training from understudy commitment in learning and substance creation to helping educators give customized content and improving understudy results. There are right now seven classifications of advancements, instruments, and techniques that driving development in training: "Buyer Technologies, Digital Strategies, Enabling Technologies, Internet Technologies, Learning Technologies, Social Media Technologies, and Visualization Technologies. Figure 15 
shows the smart classroom activities with the evolution of IoT [102].

IoT is a subcategory of Internet innovation, which bolsters instruction from multiple points of view. IoT arrangements empower instructive establishments to gather a tremendous measure of information from sensors and wearable gadgets all the more effectively and to perform significant activities dependent on this information. Such frameworks enable understudies to investigate a situation by utilizing implanted sensors, QR codes, and different advances. They can get to take in materials and other data from anyplace whenever. Educators additionally can utilize wearable gadgets and advanced cells in the homerooms to improve instructing and learning. A savvy homeroom can be characterized as a keen domain furnished with various types of equipment and programming modules [103]. Video projectors, cameras, sensors, and face acknowledgment calculations are instances of modules that screen various parameters of the physical condition or understudies' properties, for example, focus, execution, and accomplishment.

\subsection{Role of IoT in the smart retail sector}

A "smart" retail setting can be a beneficial way for a firm to generate greater customer and business value. One "smart" approach that is increasingly recognized as having the potential to create value for customers and retailers alike is the use of augmented reality in smart retail environments. Augmented reality is a smart technology that adds value to retailers by being able to influence customer engagement as well as purchasing decisions and can be varyingly used instore and out-of-store including at home. IoT is expected to play a key role as an emerging technology in the area of retail and logistics [104]. In logistics, RFID keeps track of containers, pallets, and crates. Besides, considerable advancements in IoT technologies can facilitate retailers by providing several benefits. However, IoT devices generate large amounts of data daily. Thus, powerful data analytics enables enterprises to gain insights from the voluminous amounts of data produced through IoT technologies. Applying data analytics to logistic data sets can improve the shipment experience of customers. Moreover, retail companies can earn additional profit by analyzing customer data, which can predict the trends and demands of goods. By looking into customer data, optimizing pricing plans and seasonal promotions can be planned efficiently to maximize profit [105]. The Figure 16 shows the progress in the retail sector due to the evolution of the IoT with data mining.

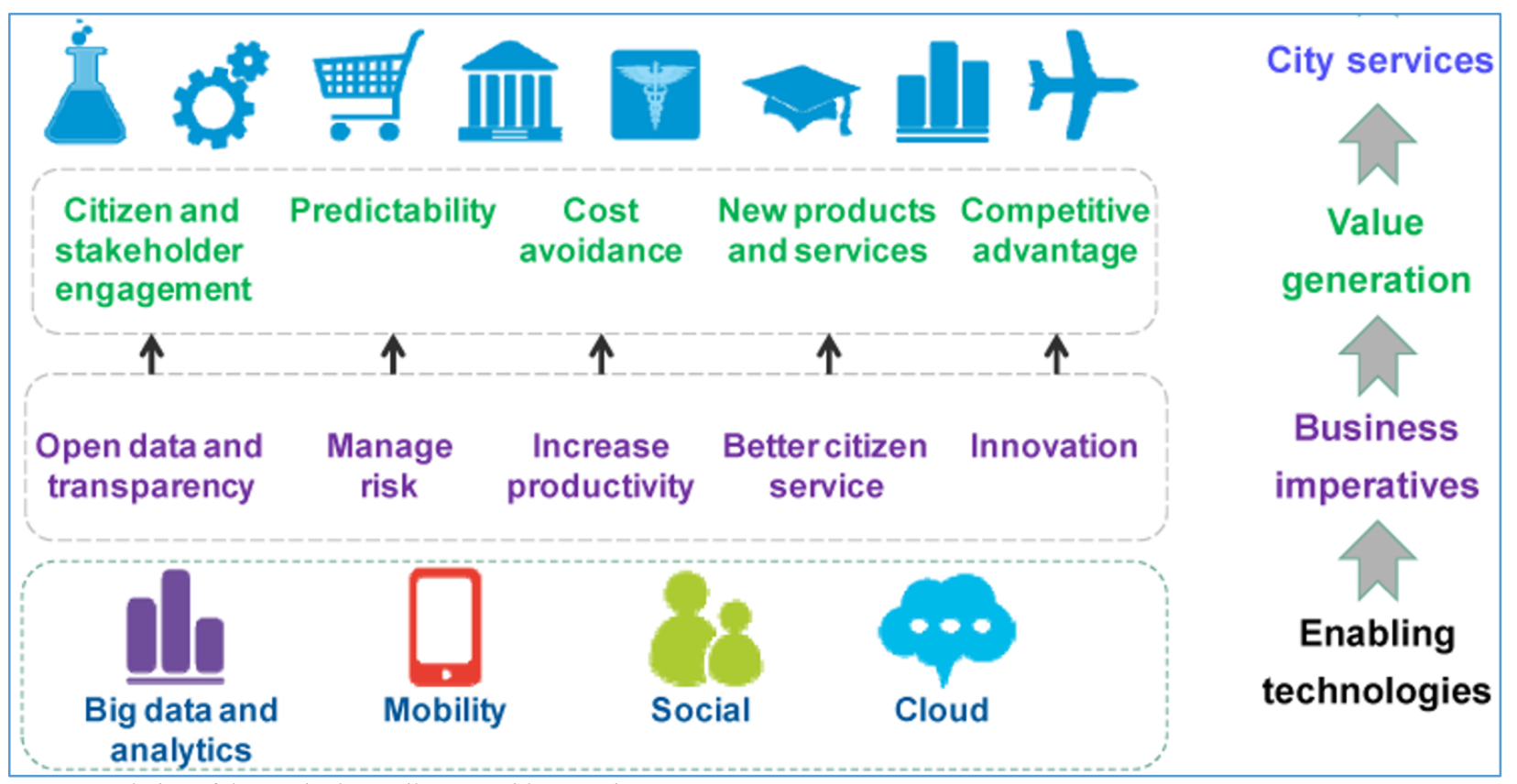

Fig. 16. Evolution of the IoT in the retail sector with step-wise progress [105]

Big IoT data analytics offers well-designed tools to process real-time big data, which produces timely results for decision making. Big IoT data exhibit heterogeneity, increasing volume, and real-time data processing features. The convergence of big data with IoT brings new challenges and opportunities to build a smart environment. Big IoT data analytics has widespread applications in nearly every industry [106]. However, the main success areas of analytics are in ecommerce, revenue growth, increased customer size, the accuracy of sale forecast results, product optimization, risk management, and improved customer segmentation.

\subsection{Role of IoT in smart home}

In a smart home environment, the price signals and demand response (DR) signals sent by grid operators need to be transmitted to the Home Energy Management System (HEMS) through smart meters, HEMS realizes power dispatch through the Home LAN (HAN) and arranges power reasonably [107]. However, this process may cause leakage or tampering of the user's power information and personal sensitive information, fine-grained data may expose users' more privacy, including working time, outgoing time, household appliances usage, etc., which will result in the users or the power company suffers property damage. Also, in the process of uploading power data, a reliable central node or gateway is used to transfer users' data, but it is difficult to find an aggregator trusted by all users, and this centralized storage mode has great risks. E.g., the failure of the central node may result in the loss of all users' data; attacks on the central node may result in data being tampered with. Based on this, there is an urgent need for a secure decentralized distributed storage method [108]. In the process of bidirectional interaction, the power data of the smart home network can be leaked in many ways. E.g., curious regional 
gateway wants to know the power data uploaded by smart meters and sells privacy for profit; attackers attack gateway to obtain users' power information; malicious unauthorized users forge user's identity to upload data and steal privacy; malicious revoked old users may use old identity information and pretend to be a legitimate user [109]. The Figure 17 shows the privacy protection scheme of a smart meter for a smart home network based on a consortium blockchain.

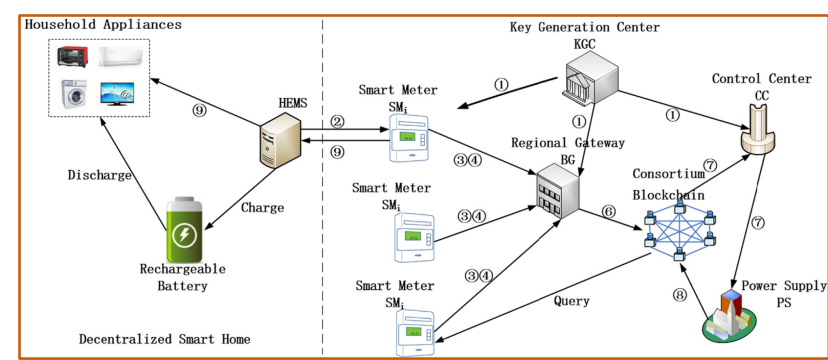

Fig. 17. Smart home application supported with blockchain technology by incorporating smart meters [109]

\subsection{Role of Smart Supply Chain}

Digitalization has changed how people communicate and interact with their surroundings. Novel technologies and gadgets: including smartphones, computers, driverless cars, and smart wearable devices have all transformed with access and disseminate information. These novelties and digital transformations affect every industry, and supply chains are no exception [110]. Digitalization in supply chains has come to encompass digital products and services as well as the handling of supply chain processes within companies undergoing these rapid changes. To benefit from the digital supply chain, it is necessary to leverage novel approaches, including digital transformation with technologies. It defines the digital supply chain as a bundle of interconnected activities, handled with novel technologies, involved in supply chain processes between suppliers and customers [111]. Digitality has changed the way that both companies and individuals interact and extremely communicate with each other. Therefore, to exploit digitalization in business, companies should consider all the necessary procedures, strategies, and tools needed to move toward the digital supply chain. The digital supply chain is defined as a bundle of interconnected activities that are involved in supply chain processes between suppliers and customers, which are handled with novel technologies [112]. The smart supply chain enables a wider availability of information and infinitely superior interactions, communication, and collaboration, which lead to improved trust, agility, and productiveness [113]. Figure 18 shows the interconnection of digitalization and smart technology with the conceptual model.

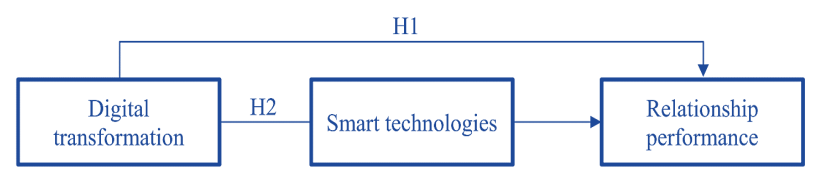

Fig 18. The layout of the smart supply chain [114]

About external collaboration activities in the digital supply chain, digital transformation can also improve customer service as it enables the collection of large amounts of data from different sources and their utilization in building strong networks between different partners. Digital transformation also makes it possible for consumers and endusers to become experts on product and service offerings.
Smart technologies extend internet-based services by incorporating technologies, such as mobile operating systems, which support several service functions, including organizational service management, collaboration management, customer service support, as well as service research and planning [114]. Additionally, to adopt smart technologies (as it is challenging and time-consuming), companies need to have certain skills and an understanding of what they seek to accomplish with the technology. Consequently, the benefits (in terms of relationship performance) will follow. From the perspective of the dynamics of the digital supply chain, it can be presented that smart technologies stimulate the growth in relationship performance [115]. This stimulation may occur when smart technologies are integrated into the current supply chain or when smart technologies entirely or partially replace the existing supply chain. From the review analysis, it is clear that cross-sectional data and a lack of longitudinal data might threaten an in-depth analysis of the data to find the required approaches in the digital supply chain [116]. Second, collecting data from a single country and analyzing them based on managerial perceptions could introduce bias and a lack of generalizability into the findings of the research. Lastly, the only endogenous variable in this study is relationship performance, which creates opportunities to expand the theoretical model of this study for other performance measures, including sales and market shares, as well as business brand and image, in terms of studying them as other endogenous variables [117].

\section{Value Added Applications}

As a result of the different Big Data projects across the world, many Big Data models, frameworks, and new technologies were created to provide more storage capacity, parallel processing, and real-time analysis of different heterogeneous sources. Besides, new solutions have been developed to ensure data privacy and security. Compared to traditional technologies, such solutions offer more flexibility, scalability, and performance. Furthermore, the cost of most hardware stores and processing solutions is continuously dropping due to sustainable technological advancement. To extract knowledge from Big Data, various models, programs, software, hardware's and technologies have been designed and proposed [118]. They try to ensure more accurate and reliable results for Big Data applications. However, in such an environment, it may be time-consuming and challenging to choose among numerous technologies. Many parameters should be considered: technological compatibility, deployment complexity, cost, efficiency, performance, reliability, support, and security risks. There exist many Big Data surveys in the literature but most of them tend to focus on algorithms and approaches used to process Big Data rather than technologies [119]. Table 2 and 3 shows the comparison of IoT Big data analytics under different use cases along with its cloud computing techniques.

Table 2. Different IoT big data analytics with its use cases

\begin{tabular}{|c|c|c|c|c|}
\hline $\begin{array}{c}\text { Application } \\
\mathbf{S}\end{array}$ & Merits & $\begin{array}{c}\text { IoT } \\
\text { devices }\end{array}$ & $\begin{array}{l}\text { Data } \\
\text { types }\end{array}$ & $\begin{array}{c}\text { Big data } \\
\text { analytics }\end{array}$ \\
\hline $\begin{array}{c}\text { Smart } \\
\text { metering } \\
{[24-29]}\end{array}$ & $\begin{array}{c}\text { Predicts } \\
\text { electricity } \\
\text { consumption }\end{array}$ & Sensors & Text & Hadoop \\
\hline $\begin{array}{c}\text { Smart } \\
\text { transportatio } \\
\mathrm{n}[42-48]\end{array}$ & $\begin{array}{l}\text { Improves } \\
\text { productive } \\
\text { performance } \\
\text { with more }\end{array}$ & $\begin{array}{c}\text { Sensors; } \\
\text { Camera } \\
\text { s }\end{array}$ & $\begin{array}{l}\text { Text, } \\
\text { Video, } \\
\text { Audio }\end{array}$ & $\begin{array}{c}\text { Hadoop, } \\
\text { Spark, } \\
\text { Hive }\end{array}$ \\
\hline
\end{tabular}




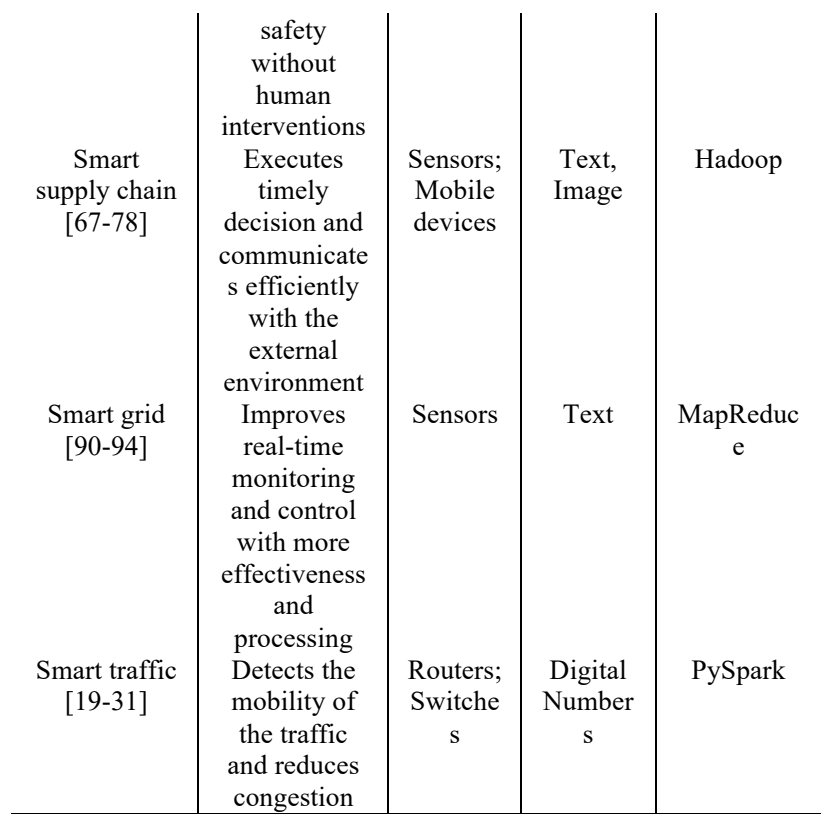

Table 3. Characterization of processing data with computing techniques

\begin{tabular}{|c|c|c|}
\hline $\begin{array}{l}\text { A smart city } \\
\text { uses case }\end{array}$ & Types of data & $\begin{array}{l}\text { Computing } \\
\text { techniques }\end{array}$ \\
\hline $\begin{array}{c}\text { Smart Traffic } \\
{[19-31]}\end{array}$ & Stream/Massive Data & Edge \\
\hline $\begin{array}{l}\text { Smart Health [76- } \\
\text { 83] }\end{array}$ & Stream/Massive Data & Edge/Cloud \\
\hline Smart & & \\
\hline $\begin{array}{c}\text { Environment } \\
{[110-119]}\end{array}$ & Stream/Massive Data & Cloud \\
\hline Smart Weather & & \\
\hline $\begin{array}{l}\text { Prediction [120- } \\
\quad 128]\end{array}$ & Stream Data & Edge \\
\hline $\begin{array}{c}\text { Smart Citizen } \\
{[10-18]}\end{array}$ & Stream Data & Cloud \\
\hline $\begin{array}{c}\text { Smart Agriculture } \\
{[130-137]}\end{array}$ & Stream Data & Edge/Cloud \\
\hline $\begin{array}{c}\text { Smart Home } \\
\text { [101-107] }\end{array}$ & $\begin{array}{c}\text { Massive/Historical } \\
\text { Data }\end{array}$ & Cloud \\
\hline $\begin{array}{c}\text { Smart Air } \\
\text { Controlling [23- } \\
27]\end{array}$ & $\begin{array}{c}\text { Massive/Historical } \\
\text { Data }\end{array}$ & Cloud \\
\hline Smart Public & & \\
\hline $\begin{array}{c}\text { Place Monitoring } \\
{[67-74]}\end{array}$ & Historical Data & Cloud \\
\hline Smart Human & & \\
\hline $\begin{array}{c}\text { Activity Control } \\
{[56-62]}\end{array}$ & Stream/Historical Data & Edge/Cloud \\
\hline
\end{tabular}

The ascent of cutting-edge innovations and its combination with different parts of our lives paved the way for the new skylines for improving the execution of structures, networks, and urban communities. Concentrating on one a significant piece of the vistas, this chapter explored the flow investigate commitments and future possibilities of IoT towards the imagined objectives of enhanced remote communication [120]. Subsequently, the current utilization of IoT towards the advancement objectives of perceptive structures was talked about, which incorporates both the scholarly world and industry use cases in the past decades. Principal criteria of practicality structures were disconnected to outline applications. Even though this is anything but a total rundown of utilizations, it can fill in as a decent beginning stage for future research in the significant regions [121]. It is inferred that present advances - equipment, programming, and figuring calculations, have just gotten a critical part of the knowledge structures improvement. Be that as it may, constant research exertion is required on IoT applications to effectively execute the possibilities of cutting-edge shrewd structures later on.

As needs are, standard specialized necessities of IoT and its incorporation necessities to the structure business were recognized. These difficulties will be significant furthermore, perfect research issues for the analysts and professionals who are keen on applying IoT ideas and framework in the structure examine territories, and further advance the advancement in both specialized and application sides of IoT [123]. Since the prime thought of IoT is to interface any individual, framework and the aggregate condition together from any area and whenever, the use of IoT will get omnipresent, and potentially the primary stream later on the advancement of savvy structures, or even to a bigger scope, for example, networks and urban areas [124]. With the top to bottom audit of stateof the-craftsmanship advancements and applications for IoT in knowledge practical oriented, this chapter hopes to give a novel and all-encompassing vision to the scholastic network and specialists who might want to dive the intensity of IoT in the various industrial application fields for additionally energizing developments.

\section{Data Mining and Machine Learning}

Mining beneficial information from multifarious sensing surrounding at diverse spatial and temporal resolutions is perplexing research problematic in artificial intelligence [125]. The main attribute risk in complex sensing locations is how to concurrently learn depictions of events and activities at multiple ranks of complexity includes events, local activities, and complex activities. Based on the present updated industrial automation strategy, machine learning techniques play a vital role in the analysis and identification of abnormalities from its historical data. Machine learning (ML) is an arena of artificial intelligence that practices statistical techniques to make computers the capability to learn with time improved performance on the desired task from data, without being overtly programmed [126]. Its intelligence confirmed by machines, in disparity to the natural intelligence revealed by humans in context, any process system that recognizes its circumstances and initiates actions that exploit its chance of controlling and identifying its reaction in an efficacious manner [127]. And also, forecasting is performed in the production, performance and economic wise to evaluate the entire throughput of the process in the future. Forecast involves merely predicting future occurrences based on the past occurred values [128]. Table 4 shows the application of data mining in various sections.

Table 4. Data mining application with computing techniques in IoT sectors

\begin{tabular}{c|c}
\hline $\begin{array}{c}\text { Machine } \\
\text { learning } \\
\text { techniques }\end{array}$ & Application sectors \\
\hline $\begin{array}{c}\text { Classification } \\
\text { [4] }\end{array}$ & $\begin{array}{c}\text { Medical imaging; Industry; Speech } \\
\text { recognition; e-governance } \\
\text { Clustering [69] } \\
\text { Healthcare; Human genetics; Market } \\
\text { analysis }\end{array}$ \\
$\begin{array}{c}\text { Association } \\
\text { rules [104] }\end{array}$ & $\begin{array}{c}\text { Bio-informatics; Industry; e- } \\
\text { governance }\end{array}$ \\
$\begin{array}{c}\text { Prediction [139] } \\
\text { Time series [97] }\end{array}$ & $\begin{array}{c}\text { Disaster management; Market } \\
\text { analytics }\end{array}$ \\
\hline
\end{tabular}


Machine learning closely overlaps with computational statistics and mathematical optimization to focus on prediction making by utilizing computers conflated with data mining [129]. Especially in the area of data analytics, machine learning is a tool used to formulate complex models and algorithms that afford predictive analytics. This analytic model provides the capability to yield consistent, accurate decisions and outcomes and expose hidden insights from historical relations and trends in data mining [130]. Figure 19 shows the machine learning algorithm for the various field with execution profiles.

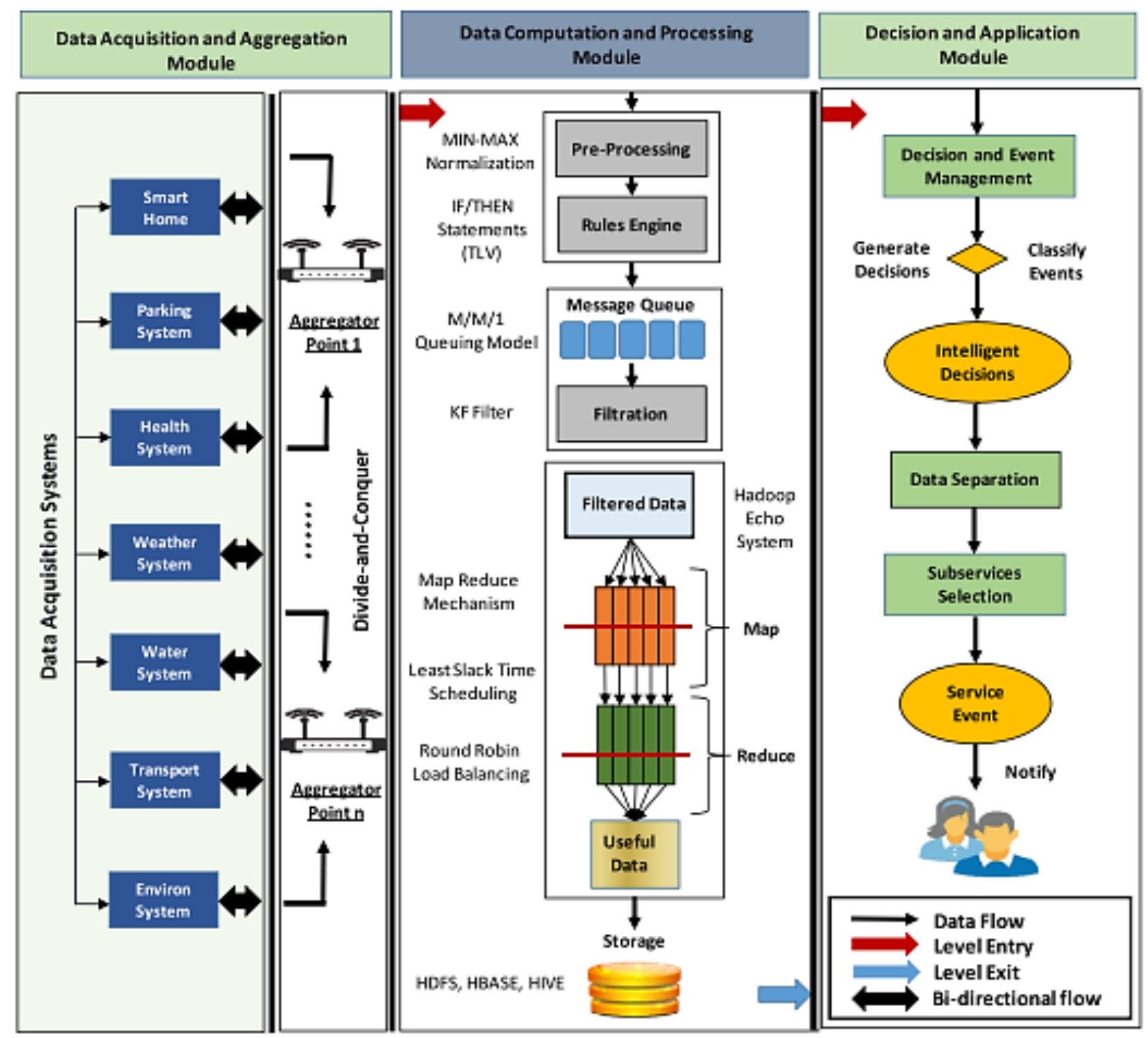

Fig. 19. Machine learning techniques with execution profile for various fields [131]

Besides, information handling and basic leadership ought to be done properly. In this manner, the amalgamation of information sources and Big Data examination is an advantageous answer to encourage ongoing the board of the brilliant city [131]. How urban communities are being concocted with brilliant gadgets utilizing enormous information is likewise featured with a few models. To perform productive Big Data investigation, it is important to total information appropriately and perform enough preprocessing before information to be prepared by the Hadoop server. The partition and-vanquish system can be extremely valuable for Big Data examination and information conglomeration. Information pre-handling (likewise known as standardization) assumes an essential job and is significant when managing parameters of various units and scales [132]. The Message Queue (MQ) offers a system that places a message over an MQ that doesn't require a moment response to carry on the preparation. Likewise, Kalman channel (KF) is utilized to more accelerate information handling and separate the important and cloud information. The commotion could be that information that isn't essential to be handled and doesn't influence the ongoing preparation. Cooperative effort (RR) is applied as a system-schedulers in handling, what's more, registering which is a structure for time-sharing, and generally broadly utilized burden adjusting approach [133]. Additionally, to accomplish ongoing preparation, planning, and burden adjusting calculation can be ideal. Also, design examining the contextual analyses utilizing a brilliant pipeline observing framework dependent on Machine Learning. The genuine acknowledgment is still in its outset, even though in the ongoing age the smart city has gotten a popular expression. Nonetheless, it is discovered that a particular zone of intrigues such as lighting, traffic blockage, and water the board is engaged by numerous the proposed work. Along these lines, there is a certainty of pragmatic perceptive urban design equipped for having clever choices to 
fortify the greatness of urban administrations [134]. Table 5 indicates the comparison of technologies involved in the smart computation platform of the current world.

Table 5. Comparison of the latest technology in different scenarios

\begin{tabular}{|c|c|c|c|}
\hline $\begin{array}{c}\text { Analytic } \\
\text { type }\end{array}$ & Implementation & $\begin{array}{c}\text { Existing } \\
\text { methodology }\end{array}$ & Merits \\
\hline $\begin{array}{l}\text { Real-time } \\
{[4-15]}\end{array}$ & $\begin{array}{l}\text { Processing massive } \\
\text { amount of data } \\
\text { from the sensors }\end{array}$ & Greenplum & $\begin{array}{c}\text { Parallel } \\
\text { processing } \\
\text { Computing } \\
\text { platforms }\end{array}$ \\
\hline $\begin{array}{l}\text { Offline } \\
\text { [69-76] }\end{array}$ & $\begin{array}{l}\text { Applications-no } \\
\text { higher requirements } \\
\text { with no response } \\
\text { time }\end{array}$ & $\begin{array}{c}\text { Scribe } \\
\text { Time tunnel }\end{array}$ & $\begin{array}{l}\text { Efficient } \\
\text { data } \\
\text { acquisition }\end{array}$ \\
\hline $\begin{array}{c}\text { Memory } \\
\text { level [103- } \\
117]\end{array}$ & $\begin{array}{c}\text { Imply where the } \\
\text { database is smaller } \\
\text { than the memory } \\
\text { level }\end{array}$ & MongoDB & Real-time \\
\hline $\begin{array}{c}\text { Intelligence } \\
\text { level [127- } \\
136]\end{array}$ & $\begin{array}{l}\text { Imply when data } \\
\text { volume suppresses } \\
\text { the memory level }\end{array}$ & $\begin{array}{l}\text { Data analysis } \\
\text { platform }\end{array}$ & $\begin{array}{l}\text { Both offline } \\
\text { and online }\end{array}$ \\
\hline $\begin{array}{l}\text { Massive } \\
\text { level [43- } \\
51]\end{array}$ & $\begin{array}{l}\text { Imply when data } \\
\text { volume surpasses } \\
\text { the entire capacity } \\
\text { of intelligence and } \\
\text { traditional data } \\
\text { management }\end{array}$ & MapReduce & $\begin{array}{l}\text { Mostly } \\
\text { switches to } \\
\text { offline }\end{array}$ \\
\hline
\end{tabular}

\section{Scope of Blockchain and Security Standards}

Blockchain is the innovation behind Bitcoin. It is an open conveyed database which holds the encoded record. Blockchain is innovation in a worldwide database that anybody, anyplace, with a web association, can utilize. In contrast to a customary database, which is claimed by focal gatherings like banks and governments, a Blockchain doesn't have a place with anybody. Figure 20 shows the block attributes in the blockchain. With a whole system caring for it, bamboozling the framework by faking report, exchanges and other data become close to outlandish. Blockchain store data for all time over a system among hubs. This isn't just decentralizing the data yet circulates it as well. Every hub in the system can store the nearby duplicate of the Blockchain framework which is intermittently refreshed to have consistency among all hubs [135]. A Blockchain is a dispersed calculation and data sharing stage that empowers various hubs that don't believe each other can take a basic leadership process. The issue is brought together framework is the single purpose of disappointment. In a decentralized framework, different organized focuses conquered the single purpose of disappointment. In a dispersed domain, each hub, on the whole, executes the activity. Every client spoke to as hub associated appropriately. Each hub kept up a duplicate of the Blockchain list which is consistently refreshed. A hub can perform distinctive movements like start a transaction, validate and exchange or perform mining.

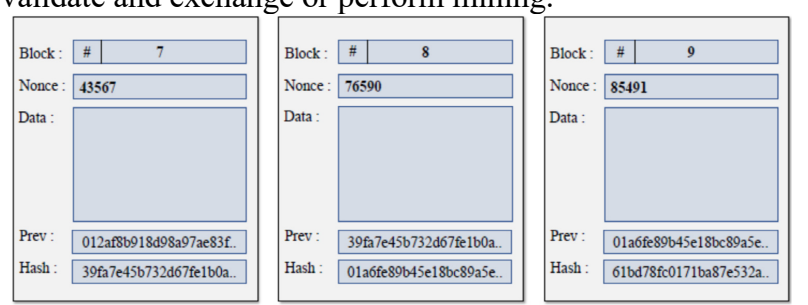

Fig. 20. Block attributes in the blockchain [136]

Blockchain's idea got from bitcoin digital currency developed as an urging innovation for shared exchange, information trustworthiness, straightforward stockpiling in a decentralized domain. The different hazards and assaults of Blockchain innovation exist in different applications some are Double spending, protection spillage, private key security, mining assault, adjusted assault. The security and protection issue of Blockchain is likewise tended to by the diverse specialist, in this area a portion of the scholarly work previously done is talked about in short. The benefits of utilizing open conveyed records (ODLs), for verifying Trust Management for verification. The information uprightness can be acknowledged through an exchange, confirmation of Blockchain properties which decreases the dangers and guarantee information honesty [136]. On proposing the thought and model of assigned verifier evidence of advantages for bitcoin trade and developed the main solid plan to understand the assigned verifier evidence of benefits for bitcoin trade by utilizing elliptic bend cryptography. Open key foundation-based structure reconciliation with Cecoin dispersed PKI conspire used to upgraded security. Cybersecurity and ensuring security are another significant issue in Blockchain usage. The flexibility of the Bitcoin biological system the unambiguousness of the Blockchain being used, the spread, and check of exchange squares. The application records mileage and GPS information of autos and verifies that on the Blockchain, which unequivocally upsets odometer extortion. Online assaults like ransomware are additionally should be tended to, in some application where arrange design manage web. The application framework must safe from outside openness generally ransomware assault can conceivable [137]. Another sort of assault is Byzantine-False Tolerant (BFT) requesting administration for the Hyperledger Fabric Blockchain stage utilizing replication library to address the security and protection issue.

\section{Conclusions}

IoT is one of the biggest sources of big data, which are rendered useless without analytics power. IoT interacts with big data when voluminous amounts of data are needed to be processed, transformed, and analyzed in high frequency. This work specifically focuses on the big data context. In the review analysis, the main objective is to investigate the recent literature on big data processing and analytics solutions for IoT. Also, to identify the numerous requirements for big data and analytics in IoT and taxonomized the literature towards the recent research uphold in the IoT for the development of smart cities in all industrial sectors. The growth rate of data production has increased drastically over the past years with the proliferation of smart and sensor devices. The interaction between IoT and big data is currently at a stage where processing, transforming and analyzing large amounts of data at a high frequency are necessary. Furthermore, big data analytics types, methods, and technologies for big data mining were presented. Some credible use cases were also provided. Also, explored the domain by discussing various opportunities brought about by data analytics in the IoT paradigm.

Current IoT and Big Data platforms are supported by various processing, analytical tools as well as dynamic visualization. Such platforms enable us to extract knowledge and value from a complex dynamic environment. They also support decision making through recommendations and automatic detection of anomalies, abnormal behavior, or new trends. Thus, this paper provides a detailed insight into the architecture, strategies, and practices that are currently followed in IoT with data computing. Despite the important 
developments in the IoT field, we can notice through our comparison of various technologies, that many shortcomings exist. Most of the time, they are related to adopted architectures and techniques. Thus, further work needs to be carried out in several areas such as data organization, domainspecific tools, and platform tools to create next-generation Big Data infrastructures. Hence, technological issues in many Big Data areas can be further studied and constitute an important research topic. Also, conclude that the existing big data solutions in the IoT paradigm are still in their infancy and the challenges associated with them must be solved in the future.

\section{Acknowledgment}

This research work is carried out under the Senior Research fellowship received from CSIR (Council for Scientific and Industrial Research) with grant no.678/08(0001)2k18 EMRI.

This is an Open Access article distributed under the terms of the Creative Commons Attribution License

\section{References}

[1] Brendan. G and Gerhard P. Hancke, Senior Member, IEEE "Introduction to Industrial Control Networks", IEEE communications surveys \& tutorials, vol. 15 , no. 2 , P 860 , the second quarter of 2013.

[2] Borgia, E. (2014). The internet of things vision: key features, applications, and open issues. Computer Communications, 54, 131.

[3] Dobre, C., \& Xhafa, F. (2014). Intelligent services for big data science. Future Generation Computer Systems, 37, 267-281.

[4] Md. Armanur Rahman, J. Hossen, Venkataseshaiah C, CK Ho, Tan Kim Geok, Aziza Sultana, Jesmeen M. Z. H., Ferdous Hossain, (2018) A Survey of Machine Learning Techniques for Self-tuning Hadoop Performance, International Journal of Electrical and Computer Engineering (IJECE)Vol.8, No.3, June2018, pp. 18541862 .

[5] Gubbi, J., Buyya, R., Marusic, S., \& Palaniswami, M. (2013). Internet of Things (IoT): a vision, architectural elements, and future directions. Future Generation Computer Systems, 29(7), 16451660 .

[6] Hollands, R. G. (2015). Critical interventions into the corporate smart city. Cambridge Journal of Regions, Economy and Society, 8(1), 61-77.

[7] El-Darymli K., F. Khan, M.H. Mohammed, Reliability modeling of wireless sensor networks for oil and gas pipelines monitoring, Sensors and Transducers Journal 108 (7) (2014) 4122-4133.

[8] Kim M., "A quality model for evaluating IoT applications," International Journal of Computer and Electrical Engineering, 8 (01) (2016) 66-70.

[9] Atzori. L, Iera. A, Morabito. G, Nitti. M (2012) The Social Internet of Things (SIoT) - when social networks meet the internet of things: concept, architecture and network characterization. Computer Networks 56(16):3594-3608.

[10] Priyanka, E.B., Maheswari, C. and Thangavel, S., 2018. IoT based field parameters monitoring and control in press shop assembly. Internet of Things, 3, pp.1-11.

[11]Ju, G., Cheng, M., Xiao, M., Xu, J., Pan, K., Wang, X., \& Shi, F. (2013). Smart transportation between three phases through a stimulus-responsive functionally cooperating device. Advanced Materials, 25(21), 2915-2919.

[12] M.R. Bashir, A.Q. Gill, Towards an iot big data analytics framework: Smart buildings systems, in: High Performance Computing and Communications; IEEE 14th International Conference on Smart City; IEEE 2nd International Conference on Data Science and Systems (HPCC/SmartCity/DSS), 2016 IEEE $18^{\text {th }}$ International Conference on, IEEE, 2016, pp. 1325-1332.

[13] C. Lee, C. Yeung, M. Cheng, Research on iot based cyber physical system for industrial big data analytics, in: Industrial Engineering and Engineering Management (IEEM), 2015 IEEE International Conference on, IEEE, 2015, pp. 1855-1859.

[14]P. Rizwan, K. Suresh, M.R. Babu, Real-time smart traffic management system for smart cities by using internet of things and big data, in: Emerging Technological Trends (ICETT), International Conference on, IEEE, 2016, pp. 1-7.

[15]Q. Zhang, X. Zhang, Q. Zhang, W. Shi, H. Zhong, Firework: Big data sharing and processing in collaborative edge environment, in: Hot Topics in Web Systems and Technologies (HotWeb), 2016 Fourth IEEE Workshop on, IEEE, 2016, pp. 20-25.

[16] M.M. Rathore, A. Ahmad, A. Paul, Iot-based smart city development using big data analytical approach, in: Automatica (ICA-ACCA), IEEE International Conference on, IEEE, 2016, pp. 1-8.
[17]B. Ahlgren, M. Hidell, E.C.-H. Ngai, Internet of things for smart cities: interoperability and open data, IEEE Internet Comput. 20 (6) (2016) 52-56.

[18] O.B. Sezer, E. Dogdu, M. Ozbayoglu, A. Onal, An extended iot framework with semantics, big data, and analytics, in: Big Data (Big Data), 2016 IEEE International Conference on, IEEE, 2016, pp. 1849-1856.

[19] B. Cheng, A. Papageorgiou, F. Cirillo, E. Kovacs, Geelytics: Geodistributed edge analytics for large scale iot systems based on dynamic topology, in: Internet of Things (WF-IoT), 2015 IEEE 2nd World Forum on, IEEE, 2015, pp. 565-570.

[20]H. Wang, O.L. Osen, G. Li, W. Li, H.-N. Dai, W. Zeng, Big data and industrial internet of things for the maritime industry in northwestern norway, in: TENCON 2015-2015 IEEE Region 10 Conference, IEEE, 2015, pp. 1-5.

[21] J.L. Pérez, D. Carrera, Performance characterization of the servioticy api: an iot-as-a-service data management platform, in: Big Data Computing Service and Applications (BigDataService), 2015 IEEE First International Conference on, IEEE, 2015, pp. 62-71.

[22] A.J. Jara, D. Genoud, Y. Bocchi, Big data for cyber physical systems: an analysis of challenges, solutions and opportunities, in: Innovative Mobile and Internet Services in Ubiquitous Computing (IMIS), 2014 Eighth International Conference on, IEEE, 2014, pp. 376-380.

[23] Z. Ding, X. Gao, J. Xu, H. Wu, Iot-statisticdb: a general statistical database cluster mechanism for big data analysis in the internet of things, in: Green Computing and Communications (GreenCom), 2013 IEEE and Internet of Things (iThings/CPSCom), IEEE International Conference on and IEEE Cyber, Physical and Social Computing, IEEE, 2013, pp. 535-543.

[24]C. Vuppalapati, A. Ilapakurti, S. Kedari, The role of big data in creating sense, an integrated approach to create next generation mobile sensor and wearable data driven electronic health record (ehr), in: Big Data Computing Service and Applications (Big Data Service), 2016 IEEE Second International Conference on, IEEE, 2016, pp. 293-296.

[25]A. Mukherjee, H.S. Paul, S. Dey, A. Banerjee, Angels for distributed analytics in iot, in: Internet of Things (WF-IoT), 2014 IEEE World Forum On, IEEE, 2014, pp. 565-570.

[26] A. Mukherjee, S. Dey, H.S. Paul, B. Das, Utilising condor for data parallel analytics in an iot context an experience report, in: Wireless and Mobile Computing, Networking and Communications (WiMob), 2013 IEEE 9th International Conference on, IEEE, 2013, pp. 325-331.

[27] M.H. Berlian, T.E.R. Sahputra, B.J.W. Ardi, L.W. Dzatmika, A.R.A. Besari, R.W. Sudibyo, S. Sukaridhoto, Design and implementation of smart environment monitoring and analytics in real-time system framework based on internet of underwater things and big data, in: Electronics Symposium (IES), 2016 International, IEEE, 2016, pp. 403-408.Rehman, M. H., Liew, C. S., Wah, T. Y., Shuja, J., \& Daghighi, B. (2015). Mining personal data using smartphones and wearable devices: a survey. Sensors, 15, 4430-4469.

[28] T. Strasser, F. Andrén, F. Lehfuss, M. Stifter and P. Palensky, "Online Reconfigurable Control Software for IEDs," in IEEE Transactions on Industrial Informatics, 9 (3 (2017) 1455-1465.

[29] W. Hu, Z. Lei, H. Zhou, G.-P. Liu, Q. Deng, D. Zhou, and Z.-W. Liu, "Plug-in free web-based 3-d interactive laboratory for control engineering education," IEEE Transactions on Industrial Electronics, 64 (5): (2017) 3808-3818. 
[30] Priyanka EB, Maheswari C, Thangavel S. Proactive Decision Making Based IoT Framework for an Oil Pipeline Transportation System. InInternational conference on Computer Networks, Big data and IoT 2018 Dec 19 (pp. 108-119). Springer, Cham.

[31] Oztemel, E., \& Gursev, S. (2020). Literature review of Industry 4.0 and related technologies. Journal of Intelligent Manufacturing, 31(1), 127-182.

[32]H. Schaffers, N. Komninos, M. Pallot, B. Trousse, M. Nilsson, A. Oliveira, Smart cities and the future internet: towards cooperation frameworks for open innovation, The Future Internet Assembly, Springer, Berlin, Heidelberg, 2011, May, pp. 431-446, , https://doi.org/10.1007/978-3-642-20898-0 31.

[33]D. Minoli, K. Sohraby, B. Occhiogrosso, IoT considerations, requirements, and architectures for smart buildings-energy optimization and next-generation building management systems, IEEE Internet Things J. 4 (1) (2017) 269-283, https://doi. org/10.1109/JIOT.2017.2647881

[34] R. C. Barros, M. F. Santos, A. F. Santos, M. F. Silva, and V. F. Vidal, "Monitoring platform, identification and control of level system based on SCADA architecture", IEEE Transactions on Industrial Electronics, 18(2018) 283-288.

[35] Abreu. M, D. Perez, K. Velasquez, M. Curado, and E. Monteiro. "A resilient Internet of Things architecture for smart cities." Annals of Telecommunications (2016): 1-12.

[36] Machado, C. G., Winroth, M. P., \& Ribeiro da Silva, E. H. D. (2020). Sustainable manufacturing in Industry 4.0: an emerging research agenda. International Journal of Production Research, 58(5), 14621484.

[37] Pereira, RI, Dupont, IM, Carvalho, PC \& Jucá, SC 2018, 'IoT embedded linux system based on Raspberry Pi applied to real-time cloud monitoring of a decentralized photovoltaic plant', Measurement, vol. 114, pp. 286-297.

[38] Gawade, P \& Meeankshi, A 2017, IOT Based Smart Public Transport System. International Journal of Scientific and Research Publications, vol.7 (7), pp.390-396.

[39]Lloret, J, Tomas, J, Canovas, A \& Parra, L 2016, 'An integrated IoT architecture for smart metering', IEEE Communications Magazine, vol. 54(12), pp. 50-57.

[40] Wazid, M., Das, A. K., Bhat, V., \& Vasilakos, A. V. (2020). LAMCIoT: Lightweight authentication mechanism in cloud-based IoT environment. Journal of Network and Computer Applications, 150, 102496 .

[41] Sanjeevi, P., T. Samraj Lawrence, V. Sathiyamoorthi, R. Manikandan, Qian Xia, and Ya Guo. "A performance-aware dynamic scheduling algorithm for cloud-based IoT applications." Computer Communications (2020).

[42] Subramaniam, T., Bhaskaran, P. (2019). Local intelligence for remote surveillance and control of flow in fluid transportation system. Advances in Modelling and Analysis C, Vol. 74, No. 1, pp. 15-21. https://doi.org/10.18280/ama_c.740102.

[43]Y.V. Pavan Kumar, R. Bhimasingu, Key Aspects of Smart Grid Design for Distribution System Automation: Architecture and Responsibilities, Procedia Technology, 21(9), 2015, pp. 352- 359, ISSN 2212-0173.

[44]Leminen, Seppo, Mika Westerlund, Mervi Rajahonka, and Riikka Siuruainen. "Towards IOT ecosystems and business models." In Internet of things, smart spaces, and next generation networking, pp. 15-26. Springer, Berlin, Heidelberg, 2012.

[45] Meribout M., "A wireless sensor network based infrastructure for real-time and online pipeline inspection", IEEE Sensor Journal, 11 (11), 2011, pp. 2966-2972.

[46] Priyanka E. B, C.Maheswari, "Parameter monitoring and control during petrol transportation using PLC based PID controller", Journal of Applied Research and Technology, 14 (5) (2016) 125131.

[47] Priyanka E.B, C. Maheswari, S. Thangavel, "Remote monitoring and control of an oil pipeline transportation system using a Fuzzy-PID controller", Flow Measurement and Instrumentation, 62 (2018) 144-151.

[48] Priyanka, E., Maheswari, C. and Thangavel, S. (2019) 'Remote monitoring and control of LQR-PI controller parameters for an oil pipeline transport system', Proceedings of the Institution of Mechanical Engineers, Part I: Journal of Systems and Control Engineering, 233(6), pp. 597-608. doi: 10.1177/0959651818803183.

[49] Priyanka, E., Maheswari, C., Ponnibala, M., \& Thangavel, S. (2019). SCADA Based Remote Monitoring and Control of Pressure \& Flow in Fluid Transport System Using IMC-PID Controller. Advances in
Systems Science and Applications, 19(3), 140-162. https://doi.org/10.25728/assa.2019.19.3.676.

[50] Priyanka, E. B., Maheswari, C., Thangavel, S. \& Bala, M. P. (2020) Integrating IoT with LQR-PID controller for online surveillance and control of flow and pressure in fluid transportation system. Journal of Industrial Information Integration, 17, 100127.

[51] Subramaniam, T., Bhaskaran, P. (2019). Local intelligence for remote surveillance and control of flow in fluid transportation system. Advances in Modelling and Analysis C, Vol. 74, No. 1, pp. 15-21. https://doi.org/10.18280/ama_c.740102.

[52] Maheswari, C, Priyanka, EB, Meenakshipriya, B. Fractional-order $\mathrm{PI}^{\lambda} \mathrm{D}^{\mu}$ controller tuned by coefficient diagram method and particle swarm optimization algorithms for $\mathrm{SO}_{2}$ emission control process. Proc IMechE, Part I: J Systems and Control Engineering 2017; 231(8): 587-599. https://doi.org/10.1177/0959651817711626

[53] Priyanka, E.B., Krishnamurthy, K. and Maheswari, C., 2016, November. Remote monitoring and control of pressure and flow in oil pipelines transport system using PLC based controller. In 2016 Online International Conference on Green Engineering and Technologies (IC-GET) (pp. 1-6). IEEE.

[54] Priyanka, E. B., Maheswari, C., \& Thangavel, S. A smart-integrated IoT module for intelligent transportation in oil industry. International Journal of Numerical Modelling: Electronic Networks, Devices and Fields, e2731.

[55]B.N. Silva, M. Khan, K. Han, Internet of things: a comprehensive review of enabling technologies, architecture, and challenges, IETE Technical Review. 35 (2) (2018) 205-220,

[56]Ali, S., Qaisar, S. B., Saeed, H., Khan, M. F., Naeem, M., \& Anpalagan, A. (2015). Network challenges for cyber physical systems with tiny wireless devices: A case study on reliable pipeline condition monitoring. Sensors, 15(4), 7172-7205.

[57] Atzori, L., Iera, A., \& Morabito, G. (2014). From" smart objects" to" social objects": The next evolutionary step of the internet of things. IEEE Communications Magazine, 52(1), 97-105.

[58] Casari, P., Castellani, A. P., Cenedese, A., Lora, C., Rossi, M., Schenato, L., \& Zorzi, M. (2009). The Wireless Sensor Networks for City-Wide Ambient Intelligence Project. Sensors, 9(6), 4056-408.

[59] Da Xu, L., He, W., \& Li, S. (2014). Internet of things in industries: A survey. IEEE Transactions on industrial informatics, 10(4), 2233-2243.

[60] Terkawi, A., \& Innab, N. (2018, April). Major Impacts of Key Reinstallation Attack on Internet of Things System. In 2018 21st Saudi Computer Society National Computer Conference (NCC) (pp. 1-6). IEEE.

[61] Kostas Psannis and Yutaka Ishibashi, Impact of Video Coding on Delay and Jitter in $3 \mathrm{G}$ Wireless Video Multicast Services, EURASIP Journal on Wireless Communications and Networking, Vol. 2006, Article ID 24616, pp. 1-7, 2006.

[62] C. Stergiou, K. E. Psannis, A. P. Plageras, Y. Ishibashi, B.-G. Kim, "Algorithms for efficient digital media transmission over IoT and cloud networking", Journal of Multimedia Information System, vol. 5, no. 1, pp. 1-10, March 2018.

[63] Vaesileios Memos, Kostas E. Psannis, Yutaka Ishibashi, Byung-Gyu Kim, Brij Gupta, An Efficient Algorithm for Media-based Surveillance System (EAMSuS) in IoT Smart City Framework, Elsevier, Future Generation Computer Systems, 2017.

[64] Lu, Z., Wang, N., Wu, J., \& Qiu, M. (2018). IoTDeM: An IoT Big Data-oriented MapReduce performance prediction extended model in multiple edge clouds. Journal of Parallel and Distributed Computing, 118, 316-327.

[65] C. Stergiou, K. E. Psannis, B.-G. Kim, B. Gupta, "Secure integration of IoT and Cloud Computing", Elsevier,Future Generation $\begin{array}{lll}\text { Computer } & \text { Systems, } & \text { December }\end{array}$ 2016.http://doi.org/10.1016/j.future.2016.11.031. 5.

[66] A. P. Plageras, K. E. Psannis, C. Stergiou, H. Wang, B. B. Gupta, "Efficient IoT-based sensor BIG Data collection-processing and analysis in Smart Buildings", Future Generation Computer Systems, vol. 82, pp. 349-357, May 2018.

[67] C. Stergiou, K. E. Psannis, B.-G. Kim, B. Gupta, "Secure integration of IoT and Cloud Computing", Elsevier, Future Generation Computer Systems, vol. 78, part 3, pp. 964-975, January 2018.

[68] Sabina Jeschke, Christian Brecher, Houbing Song, and Danda Rawat, Industrial Internet of Things: Cyber manufacturing Systems. ISBN: 978-3-319-42558-0, Cham, Switzerland: Springer, 2017, pp. 1-715. 
[69] Guido Dartmann, Houbing Song, and Anke Schmeink. Big Data Analytics for Cyber-Physical Systems: Machine Learning for the Internet of Things. ISBN: 9780128166376. Elsevier, 2019, pp. 1360 .

[70]Houbing Song, Danda Rawat, Sabina Jeschke, and Christian Brecher. Cyber-Physical Systems: Foundations, Principles and Applications. ISBN: 978-0-12-803801-7. Boston, MA: Academic Press, 2016, pp. 1-514.

[71] Gou, Q., Yan, L., Liu, Y., \& Li, Y. (2013, August). Construction and strategies in IoT security system. In 2013 IEEE international conference on green computing and communications and IEEE internet of things and IEEE cyber, physical and social computing (pp. 1129-1132). IEEE.

[72]Houbing Song, Ravi Srinivasan, Tamim Sookoor, Sabina Jeschke, Smart Cities: Foundations, Principles and Applications. ISBN: 9781-119-22639-0, Hoboken, NJ: Wiley, 2017, pp.1-906.

[73] G. Broll, E. Rukzio, M. Paolucci, M. Wagner, A. Schmidt, H. Hussmann, Perci: pervasive service interaction with the internet of things, IEEE Internet Comput. 13(6) (2009) 74-81, https://doi.org/10.1109/MIC.2009.120.

[74] M. Darianian, M.P. Michael, Smart home mobile RFID-based internet-of-things systems and services, 2008 International Conference on Advanced Computer Theory and Engineering, 2008, pp. 116-120 (IEEE), https://doi.org/10.1109/ ICACTE.2008.180.

[75]A. Whitmore, A. Agarwal, L. Da Xu, The internet of things - a survey of topics and trends, Inf. Syst. Front. 17 (2) (2015) 261-274, https://doi.org/10.1007/s10796-014-9489-2.

[76] Maheswari, C., E. B. Priyanka, S. Thangavel, and P. Parameswari. "Development of Unmanned Guided Vehicle for Material Handling Automation for Industry 4.0."

[77] Pratheep, V. G., Priyanka, E. B., Thangavel, S., Gousanal, J. J., Antony, P. B., \& Kavin, E. D. (2020). Investigation and analysis of corn cob, coir pith with wood plastic composites. Materials Today: Proceedings.

[78] Pratheep, V. G., Priyanka, E. B., \& Prasad, P. H. (2019, October). Characterization and Analysis of Natural Fibre-Rice Husk with Wood Plastic Composites. In IOP Conference Series: Materials Science and Engineering (Vol. 561, No. 1, p. 012066). IOP Publishing.

[79] Shankar, R. K., Priyanka, E. B., \& Saravanan, B. (2015). Performance analysis of gasoline direct injection in two-stroke spark-ignition engines. Int J Adv Res Electr Electron Instrum Eng, 4, 4940-4947.

[80] Kaliyannan, G. V., Palanisamy, S. V., Priyanka, E. B., Thangavel, S., Sivaraj, S., \& Rathanasamy, R. (2020). Investigation on sol-gel based coatings application in energy sector-A review. Materials Today: Proceedings.

[81] Maheswari, C., Priyanka, E.B., Thangavel, S., Ram Vignesh SV., Poongodi, C., Multiple regression analysis for the prediction of extraction efficiency in mining industry with industrial IoT. Prod. Eng. Res. Devel. (2020). https://doi.org/10.1007/s11740-020$\underline{00970-\mathrm{Z}}$

[82]I. Gartner, Gartner Says 6.4 Billion Connected "Things" Will Be in Use in 2016, Up 30 Percent From 2015, http://www.gartner.com/newsroom/id/3165317, Accessed date: 23 April 2018.

[83]D. Evans, The internet of things: how the next evolution of the internet is changing everything, CISCO White Paper, 1(2011) 2011, pp. $1-11$.

[84]M.R. Abdmeziem, D. Tandjaoui, I. Romdhani, Architecting the internet of things: state of the art, Robots and Sensor Clouds, Springer, Cham, 2016, pp. 55-75, ,https://doi.org/10.1007/978-3319-22168-7_3.

[85]A. Al-Fuqaha, M. Guizani, M. Mohammadi, M. Aledhari, M. Ayyash, Internet of things: a survey on enabling technologies, protocols, and applications, IEEE Commun. Surv. Tutorials 17 (4) (2015) https://doi.org/10.1109/COMST.2015.2444095.

[86] Knud Lasse Lueth, The 10 most popular Internet of Things applications right now, https://IoT-analytics.com/10-internet-ofthings-applications/, (2015), Accessed date: 16 June 2018.

[87] M. Alaa, A.A. Zaidan, B.B. Zaidan, M. Talal, M.L.M. Kiah, A review of smart home applications based on Internet of Things, J. Netw. Comput. Appl. 97 (2017) 48-65, https://doi.org/10.1016/j.jnca.2017.08.017.

[88] J. Lin, W. Yu, N. Zhang, X. Yang, H. Zhang, W. Zhao, A survey on internet of things: architecture, enabling technologies, security and privacy, and applications, IEEE Internet Things J. 4 (5) (2017) 1125 1142, https://doi.org/10.1109/JIOT.2017.2683200.
[89] A.J. Trappey, C.V. Trappey, U.H. Govindarajan, A.C. Chuang, J.J. Sun, A review of essential standards and patent landscapes for the internet of things: a key enabler for industry 4.0, Adv. Eng. Inform. 33 (2017) 208-229, https://doi.org/10.1016/j.aei.2016.11.007.

[90]B.N. Silva, M. Khan, K. Han, Internet of things: a comprehensive review of enabling technologies, architecture, and challenges, IETE Tech. Rev. $35 \quad$ (2) (2018) 205-220, https://doi.org/10.1080/02564602.2016.1276416.

[91] M. Jia, R.S. Srinivasan, Occupant behavior modeling for smart buildings: a critical review of data acquisition technologies and modeling methodologies, Winter Simulation Conference (WSC), 2015, 2015, December, pp. 3345-3355 (IEEE), https://doi.org/10.1109/WSC.2015.7408496.

[92] G. Demiris, M. Skubic, M. Rantz, J. Keller, M. Aud, B. Hensel, Z. $\mathrm{He}$, Smart home sensors for the elderly: a model for participatory formative evaluation, Hum. Comput. Interact. 6 (2006) 7-10.

[93] Thomas, R. J., Anderson, N. A., Donaldson, S. G., \& Behar, M. A. (2009). U.S. Patent No. 7,567,844. Washington, DC: U.S. Patent and Trademark Office.

[94] Y. Ham, K.K. Han, J.J. Lin, M. Golparvar-Fard, Visual monitoring of civil infrastructure systems via camera-equipped Unmanned Aerial Vehicles (UAVs): a review of related works, Vis. Eng. 4 (1) (2016) 1, https://doi.org/10.1186/s40327-015-0029-z.

[95]D. Ding, R.A. Cooper, P.F. Pasquina, L. Fici-Pasquina, Sensor technology for smart homes, Maturitas 69 (2) (2011) 131-136, https://doi.org/10.1016/j.maturitas.2011.03.016.

[96]Z. Ren, C.J. Anumba, J. Tah, RFID-facilitated construction materials management (RFID-CMM)-a case study of water-supply project, Adv. Eng. Inform. $25 \quad$ (2) (2011)198-207, https://doi.org/10.1016/j.aei.2010.02.002.

[97] Bhaskaran, P. E., Chennippan, M., \& Subramaniam, T. (2020). Future prediction \& estimation of faults occurrences in oil pipelines by using data clustering with time series forecasting. Journal of Loss Prevention in the Process Industries, 66, 104203. https://doi.org/10.1016/j.jlp.2020.104203

[98] Priyanka, E. B., \& Subramaniam, T. (2018). Fuzzy Logic Forge Filter Weave Pattern Recognition Analysis on Fabric Texture. International Journal of Electrical and Electronic Science, 5(3), 63-70.

[99]Elhoseny, M., Ramírez-González, G., Abu-Elnasr, O. M., Shawkat, S. A., Arunkumar, N., \& Farouk, A. (2018). Secure medical data transmission model for IoT-based healthcare systems. Ieee Access, 6, 20596-20608.

[100] Priyanka, E. B., \& Thangavel, S. (2018). Optimization of LargeScale Solar Hot Water System Using Non-traditional Optimization Technique. J Environ Sci Allied Res, 2018, 05-14.

[101] Bagheri, M., \& Movahed, S. H. (2016, November). The effect of the Internet of Things (IoT) on education business model. In 2016 12th International Conference on Signal-Image Technology \& Internet-Based Systems (SITIS) (pp. 435-441). IEEE.

[102] Priyanka, E. B., \& Thangavel, S. (2018). Advanced Enhancement Model of Bionics Fish Cilia MEMS Vector HydrophoneSystematic Analysis Review. International Journal of Applied Physics, 3.

[103] W.S. Jang, W.M. Healy, M.J. Skibniewski, Wireless sensor networks as part of a web-based building environmental monitoring system, Autom. Constr. 17 (6) (2008) 729-736, https://doi.org/10.1016/j.autcon.2008.02.001.

[104] Jankatti, S., Raghavendra, B. K., Raghavendra, S., \& Meenakshi, M. (2020). Performance evaluation of Map-reduce jar pig hive and spark with machine learning using big data. International Journal of Electrical and Computer Engineering, 10(4), 3811.

[105] Caro, F., \& Sadr, R. (2019). The Internet of Things (IoT) in retail: Bridging supply and demand. Business Horizons, 62(1), 47-54.

[106] A. Zanella, N. Bui, A. Castellani, L. Vangelista, M. Zorzi, Internet of things for smart cities, IEEE Internet Things J. 1 (1) (2014) $22-$ 32, https://doi.org/10.1109/JIOT.2014.2306328.

[107] S.T. Bushby, BACnetTM: a standard communication infrastructure for intelligent buildings, Autom. Constr. 6 (5) (1997) 529-540, https://doi.org/10.1016/S0926-5805(97)000290.

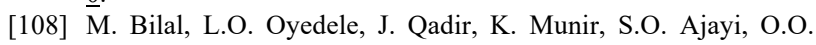
Akinade, H.A. Owolabi, H.A. Alaka, M. Pasha, Big data in the construction industry: a review of present status, opportunities, and future trends, Adv. Eng. Inform. 30 (3) (2016) 500-52, https://doi.org/10.1016/j.aei.2016.07.001.

[109] Memon, Raheel Ahmed, Jianping Li, Junaid Ahmed, Muhammad Irshad Nazeer, Muhammad Ismail, and Khursheed Ali. "Cloudbased vs. blockchain-based IoT: a comparative survey and way 
forward." Frontiers Inf. Technol. Electron. Eng. 21, no. 4 (2020): 563-586.

[110] Janak, J., Schulzrinne, H., 2016. Framework for rapid prototyping of distributed IoT applications powered by WebRTC. In: Proceedings of the IEEE Conference on Principles, Systems and Applications of IP Telecommunications, Chicago, IL, USA.

[111] Jara, A., Zamora, M., Skarmeta, A., Glowbal, I.P., 2012. An adaptive and transparent IPv6 integration in the Internet of things. Mob. Info. Syst. 8, 177-197.

[112] Jararweh, Y., Doulat, A., AlQudah, O., Ahmed, E., Al-Ayyoub, M., Benkhelifa, E., 2016.The future of mobile cloud computing: integrating cloudlets and mobile edge computing. In: Proceedings of the 23rd International Conference on Telecommunications, Thessaloniki, Greece.

[113] Jere, A., Meza, M., Marusic, B., Dobravec, S., Finkst, T., Tasic, J., 2003. Peer to peer search engine and collaboration platform based on JXTA protocol. In: Proceedings of the IEEE Region 8 EUROCON 2003 Computer as a Tool, Ljubljana, Slovenia, pp.256-260.

[114] Wu, Lifang, Xiaohang Yue, Alan Jin, and David C. Yen. "Smart supply chain management: a review and implications for future research." The International Journal of Logistics Management (2016).

[115] Nitti, M., Pilloni, V., Colistra, G., Atzori, L., 2015. The virtual object as a major element of the Internet of things: a survey. IEEE Commun. Surveys Tutorials 18 (2),1228-1240.

[116] N. Li, B. Becerik-Gerber, L. Soibelman, B. Krishnamachari, Comparative assessment of an indoor localization framework for building emergency response, Autom. Constr. 57 (2015) 42-54, https://doi.org/10.1016/j.autcon.2015.04.004.

[117] Y. Jie, J.Y. Pei, L. Jun, G. Yun, X. Wei, Smart home system based on IOT technologies, 2013 International Conference on Computational and Information Sciences, 2013, pp. 1789-1791 (IEEE), https://doi.org/10.1109/ICCIS.2013.468.

[118] V. Fabi, R.V. Andersen, S.P. Corgnati, B.W. Olesen, M. Filippi, Description of occupant behaviour in building energy simulation: state-of-art and concepts for improvements, 12th Conference of International Building Performance Simulation Association, 1416 2011, November, pp. 2882-2889.

[119] M. Jia, R.S. Srinivasan, A.A. Raheem, From occupancy to occupant behavior: an analytical survey of data acquisition technologies, modeling methodologies and simulation coupling mechanisms for building energy efficiency, Renew. Sust. Energ. Rev. 68 (2017) 525-540, https://doi.org/10.1016/j.rser.2016.10.011.

[120] F. Zafari, I. PapapanagIoTou, K. Christidis, Microlocation for internet-of-thingsequipped smart buildings, IEEE Internet Things $\begin{array}{llll}\text { J. } & 3 & \text { (1) } & \text { (2016) }\end{array}$ https://doi.org/10.1109/JIOT.2015.2442956.

[121] C. Gomez, J. Oller, J. Paradells, Overview and evaluation of bluetooth low energy: an emerging low-power wireless technology, Sensors 12 (12) (2012) 11734-11753, https://doi.org/10.3390/s120911734.

[122] Ra, M., Priyantha, B., Kansal, A., Liu, J., 2012. Improving energy efficiency of personal sensing applications with heterogeneous multi-processors. In: Proceedings of the ACM Conference on Ubiquitous Computing, Pittsburgh, PA, USA, pp. 1-10.
[123] Rabin, M., 1981. Fingerprinting by Random Polynomials. Technical report TR-15-81. Department of Computer Science, Harvard University, Cambridge, MA, USA.

[124] Radoev, M., 2017. A comparison between characteristics of NoSQL databases and traditional databases. Comput. Sci. Info. Technol. 5 (5), 149-153.

[125] Raei, H., Yazdani, N., Shojaee, R., 2017. Modeling and performance analysis of cloudlet in mobile cloud computing. Perform. Evaluat. 107, 34-53.

[126] Rahmani, A., Gia, T., Negash, B., Anzanpour, A., Azimi, I., Jiang, M., Liljeberg, P., 2018. Exploiting smart e health gateways at the edge of healthcare Internet-of-things: a fog computing approach. Future Generat. Comput. Syst. 78, 641-658.

[127] Rajaram, K., Susanth, G., 2017. Emulation of IoT gateway for connecting sensor nodes in heterogeneous networks. In: Proceedings of the IEEE International Conference on Computer, Communication, and Signal Processing, Chennai, India.

[128] Ramlee, R., Othman, M., Leong, M., Ismail, M., Ranjit, S., 2013. Smart home system using Android application. In: Proceedings of the International Conference of Information and Communication Technology, Bandung, Indonesia, pp. 277-280.

[129] Razafimandimby, C., Loscrí, V., Vegni, A., Neri, A., 2017. A Bayesian and smart gateway-based communication for noisy IoT scenario. In: Proceedings of the International Conference on Computing, Networking and Communications, Santa Clara, USA.

[130] Narayanan, D., Flinn, J., Satyanarayanan, M., 2000. Using history to improve mobile application adaptation. In: Proceedings of the 3rd IEEE Workshop on Mobile Computing Systems and Applications, Los Alamitos, CA, USA, pp. 31-40.

[131] Hindy, H., Bayne, E., Bures, M., Atkinson, R., Tachtatzis, C., \& Bellekens, X. (2020). Machine Learning Based IoT Intrusion Detection System: An MQTT Case Study. arXiv preprint arXiv:2006.15340.

[132] Narendra, N., Koorapati, K., Ujja, V., 2015. Towards cloud-based decentralized storage for Internet of things data. In: Proceedings of the 2015 IEEE International Conference on Cloud Computing in Emerging Markets, Bangalore, India, pp. 160-168.

[133] Zhou, Qiheng, Huawei Huang, Zibin Zheng, and Jing Bian. "Solutions to scalability of blockchain: A survey." IEEE Access 8 (2020): 16440-16455.

[134] Ramírez-Gallego, Sergio, Alberto Fernández, Salvador García, Min Chen, and Francisco Herrera. "Big Data: Tutorial and guidelines on information and process fusion for analytics algorithms with MapReduce." Information Fusion 42 (2018): 5161.

[135] Yuvaraj, S., \& Sangeetha, M. (2016, March). Smart supply chain management using internet of things (IoT) and low power wireless communication systems. In 2016 International Conference on Wireless Communications, Signal Processing and Networking (WiSPNET) (pp. 555-558). IEEE.

[136] Li, Xiaoqi, Peng Jiang, Ting Chen, Xiapu Luo, and Qiaoyan Wen. "A survey on the security of blockchain systems." Future Generation Computer Systems 107 (2020): 841-853.

[137] Abdel-Basset, Mohamed, Gunasekaran Manogaran, and Mai Mohamed. "Internet of Things (IoT) and its impact on supply chain: A framework for building smart, secure and efficient systems." Future Generation Computer Systems 86 (2018): 614628.

$\begin{array}{cc}\begin{array}{c}\text { Nomenclature } \\ \text { Notations }\end{array} & \text { Abbreviations } \\ \text { IoT } & \text { Internet of Things } \\ \text { AI } & \text { Artificial Intelligence } \\ \text { ITU } & \text { International Telecommunication Union } \\ \text { MQTT } & \text { Message Queue Telemetry Transport } \\ \text { DDS } & \text { Data Distribution Service } \\ \text { DBMS } & \text { Database Management Systems } \\ \text { EHR } & \text { Electronic Health Records } \\ \text { IOUT } & \text { Internet of Underwater Things } \\ \text { ICT } & \text { Information and Communications } \\ \text { DSL } & \text { Technology } \\ \text { PSTN } & \text { Digital Subscriber Line } \\ \text { iDEN } & \text { Public Switched Telephone Network } \\ \text { CDMA } & \text { Code Division Multiple Access } \\ \text { TDMA } & \text { Time Division Multiple Access }\end{array}$

Notations
PID
WSN
IT
VN
LTE
SLN
DDB
RN
BBN
QoS
TLP
DR
HEMS
HAN

\author{
Abbreviations \\ Proportional-Integral-Derivative \\ Wireless Sensor Network \\ Information Technology \\ vehicle hub \\ Long Term Evolution \\ Server Chief Hub \\ Disseminated Database \\ Root Hub \\ Body-to-Body Network \\ Quality of Service \\ Transmit Level Parallelism \\ Demand Response \\ Home Energy Management System
}


E.B. Priyanka and S. Thangavel/Journal of Engineering Science and Technology Review 13 (4) (2020) 1 - 21

SCADA

IaaS

PaaS

SaaS

RFID
Supervisory Control \& Data Acquisition Infrastructure as a Service

Platform as a Service

Software as a Service

Radio Frequency Identification
ML

KF

MQ

ODL

BFT
Machine Learning

Kalman Channel

Message Queue

Open Conveyed Records

Byzantine-False Tolerant 